\title{
Natura ed origini della Bibliografia: una prospettiva disciplinare per la contemporaneità
}

\author{
Nature and origin of Bibliography: disciplinary perspective to contemporaneity
}

\begin{abstract}
Fiammetta Sabba
PhD in Scienze Bibliografiche, Archivistiche, Documentarie e per il Restauro e la Conservazione dei beni librari e archivistici - Università degli Studi di Udine. Professore Universitario Associato presso il Dipartimento di Beni Culturali - Università degli Studi di Bologna.

E-mail: fiammetta.sabba@unibo.it
\end{abstract}

\begin{abstract}
Riassunto
L'articolo propone lo studio della Bibliotheca Universalis di Conrad Gesner per mezzo della tipologia delle fonti di informazione usate dall' autore nella sua composizione e della loro rappresentatività bibliografica in relazione alla cultura scritta disponibile all' epoca. Con questa base, propone la misurazione del grado di 'universalità' dell' opera nella prospettiva della meta desiderata e degli strumenti a sua disposizione. Per questo, se elabora un percorso di revisione delle fonti usate da Gesner, che variano tra cataloghi editoriali, cataloghi di biblioteche oltre che bibliografie propriamente dette, fonti ottenute, nella maggior parte dei casi, durante i viaggi fatti dall' autore. Si distacca, in questo modo, nella ricostituzione di questo percorso di ricerca di Gesner, l' attualità del suo progetto bibliografico, che si basa nel principio di registrare il patrimonio scritto, di classificarlo e identificare quello che ancora bisognava rendere pubblico. Inoltre, si sviluppa una discussione che espone le affinità e i parallelismi tra le necessita di una costruzione 'semantica' dell' informazione oggigiorno e l' attualità, a questo fine, del Metodo Gesneriano.
\end{abstract}

Parole-chiave: Bibliografia - Storia e Teoria. Conrad Gesner (1516-1565). Bibliotheca Universalis (1545). Metodo Gesneriano. Pandectae (1548-1549).

\begin{abstract}
The paper proposes the study of Bibliotheca Universalis of Conrad Gessner through the typology of information sources used by the author in its composition and its bibliographical representation in relation to written culture available at the time. On that basis, the paper proposes to measure the degree of 'universality' of the work in view of the desired goal and the instruments at their disposal. For this, it prepares a route review of the sources used by Gesner ranging from publishing catalogs, library catalogs, and also actual references, sources obtained, most often over trips made by the author. Stands thus in the reconstruction of this Gesner route research, the relevance of his bibliographic project, based on the principle of recording the written heritage, to classify it and identify what still needed to be made public. It develops also a discussion that aims to show affinities and parallels between the needs of a 'semantic' construction information in our times and the actuality of Gesnerian method therefore.
\end{abstract}

Keywords: Bibliography - History and Theory. Conrad Gesner (1516-1565). Bibliotheca Universalis (1545). Gesnerian Method. Pandectae (1548-1549).

InCID: R. Ci. Inf. e Doc., Ribeirão Preto, v. 7, n. esp., p. 99-133, ago. 2016.

DOI: 10.11606/issn.2178-2075.v7iespp99-133 


\section{Introduzione}

Questo contributo è l'elaborazione scritta della "Conferência de Encerramento" che ho tenuto il 4 dicembre 2015 presso la Biblioteca della Facoltà di Economia e Amministrazione della Università di San Paolo (FEA-USP) in occasione del II Seminario internazionale "A Arte da Bibliografia: História, Natureza e Relaçõnes (Inter)Disciplinares" realizzato in seno al Programma di Post-laurea in Scienza dell'Informazione della Scuola di Comunicazioni ed Arti della stessa Università ospitante.

Nell'intitolazione del Seminario è stato utilizzato il termine di 'Arte' in riferimento alla Bibliografia; si è trattato di un accostamento criptico che ha finito però per svelarsi e chiarirsi nel corso delle due giornate convegnistiche: 'Arte bibliografica' come tecnica, ma anche tecnologia, ed ancora soprattutto come conoscenze, competenze, sapienze, espedienti e strategie della Bibliografia in rapporto alla tradizione scritta, comprendendovi anche sezioni di quella segnica e di quella simbolica, per una interpretazione storica ed insieme avveniristica del mondo dell'informazione.

Il concetto di 'informazione' è a sua volta complesso ed esteso, e il suo valore dipende dal punto di vista delle discipline e dei campi che lo usano, lo trattano e lo analizzano, come gli ambiti informatico, cibernetico, filosofico, giornalistico, artistico, socioculturale...

Attenzione va fatta a parlare di dati come informazioni, ciò vale sempre e tanto più nel nostro campo delle 'comunicazioni documentarie'. Affinché un dato o gruppi di dati si trasformino in informazione la loro ricezione deve infatti avvenire da parte di una struttura già sufficientemente complessa e attrezzata a riceverli e ad elaborarli. I dati si trasformano in informazione dunque solo nelle condizioni in cui possono contribuire ad innalzare ancora il livello di complessità di cui quella struttura ricevente è già portatrice.

La Scienza dell'informazione è una disciplina, interessante, ricca, attuale e necessaria, ma non è una scienza dal punto di vista costituzionale perché non ha un suo proprio protocollo epistemologico e metodologico che permetta comparazioni. Essa attinge invece dai risultati di altre discipline per comporre le sue teorie e per costruire le sue prospettive. Ciò ci induce a sostenere che essa non è una scienza di per sé, ma lo diventa in quanto assume una natura transdisciplinare che le impone un metodo non tanto di consolidamento di se stessa quanto di coesione e di amalgama di altre scienze, e pertanto possiamo definire più correttamente la Scienza dell'informazione una scienza delle comunicazioni. 
Questa premessa ci introduce alla riflessione sulla Bibliografia, sul suo ruolo come disciplina e sui suoi presupposti concettuali in quanto 'Scienza delle comunicazioni registrate'. Dal punto di vista bibliografico ci interessa infatti quella informazione che è legata alla produzione letteraria e scientifica scritta. Ciò, come già per la Scienza dell'informazione, ci costringe a fare una valutazione epistemologica, e a chiederci se i temi affrontati dalla Bibliografia non abbiano invece diritto d'essere trattati dai settori scientifici della Storia e della Storia della Letteratura, o se le 'Discipline del libro e del documento' abbiano dunque davvero una solida tradizione e sufficienti motivi per esistere autonomamente dal punto di vista sia scientifico che accademico.

\section{Natura e ruolo disciplinare della Bibliografia}

Per comprendere il ruolo della Bibliografia è necessario comprendere prima che natura essa abbia, sia attraverso il suo rapporto con la biblioteca come concetto teorico e come sistema gestionale, che mediante il suo legame con la Storia della Bibliografia e con la Storia delle biblioteche.

I termini di 'biblioteca' e di 'bibliografia' hanno la stessa radice semantica, ed è quella contenuta nelle parole greche ó $\beta i ́ \beta \lambda o \varsigma-o v=$ corteccia, libro, e $\tau$ ó $\beta \imath \beta \lambda$ íov-ov = carta, scritto, opera, libro; mentre si differenziano per la desinenza diversa derivata dalle parole $\eta \dot{\eta} \theta \dot{\eta} \kappa \eta-\eta \varsigma=$ cassa, scrigno, contenitore, e $\eta \dot{~} \gamma \rho \alpha \varphi \eta ́-\eta \varsigma$ = scrittura. Con la parola latina 'bibliotheca' almeno fino al XVII secolo si intendeva sia una 'libraria' che un 'catalogus', in quanto si trattava di un contenitore di libri sia se essi fossero presenti fisicamente (ordinati negli scaffali e nei palchetti della biblioteca a fini consultativi) sia presenti virtualmente (attraverso una loro segnalazione a scopo informativo, secondo un ordine alfabetico o sistematico, in un catalogo o in un repertorio bibliografico).

In questa sovrapposizione si ritrova paradossalmente l'essenza metafisica della Bibliografia, che si occupa infatti di accogliere e ordinare i connotati letterari e gli elementi semantici dei documenti generati dalle altre discipline, nell'ambito delle quali verranno poi successivamente riutilizzati. La Bibliografia, pertanto, consiste in una metastruttura, ed è essa stessa una metadisciplina che si fa ancilla e domina rispetto a tutte le altre categorie disciplinari: utilizza i prodotti scritti nell'ambito delle altre discipline e li restituisce loro in una forma organizzata, in modo da garantirne la rintracciabilità, la permanenza segnaletica, e laddove 
possibile la fruizione.

Per questa attività organizzativa la bibliografia si serve degli 'indici' che sono appunto quei connotati già detti, inquadrati in schemi categoriali che mediano tra le esigenze esplicite, implicite e latenti dell'utente, o dei gruppi di utenti, e le offerte informazionali documentarie. I piani di azione sono quello letterario-autoriale (autori, opere e edizioni), e quello semanticoconcettuale (soggetti, materie, classi, ambiti disciplinari). Si tratta dell'impostazione che ritroviamo nei Functional Requirement for Bibliographic Records (FRBR) con cui l'International Federation of Library Associations and Institutions (IFLA) dal 1998 si è impegnata a adeguare alle nuove esigenze dell'utenza le norme catalografiche formulate nel 1961 dai Principi di Parigi.

- Entità 1. gruppo - opera

$$
\begin{aligned}
& \text { - espressione } \\
& \text { - manifestazione } \\
& \text { - item }
\end{aligned}
$$

- Entità 2. gruppo - persone/enti responsabili (nella creazione, realizzazione, distribuzione e gestione delle entità del primo gruppo)

$$
\begin{array}{cl}
\text { Entità 3. gruppo } & \text { - concetto } \\
\text { (soggetti delle opere) } & \text { - oggetto } \\
& \text { - evento } \\
& \text { - luogo }
\end{array}
$$

Gli indici si configurano in questo modo come il prodotto e lo strumento delle attività di mappatura e organizzazione della Bibliografia, e la Bibliografia può venire così definita anche 'Scienza delle comunicazioni mediate da indici'.

La Bibliografia si dirama però in due filoni, uno è quello attuale e uno quello storico, che hanno entrambi un compito primario per la conoscenza: il primo ha un ruolo fondamentale nella permanenza delle memorie scritte, che sono la garanzia della civiltà umana; il secondo ne interpreta le tappe teoretiche ed evolutive disciplinari, spiegando le 'naturali selezioni' avvenute sia per i documenti che per le mappe teoretiche in seno ad ogni epoca o territorio culturale. Questo significa che esiste anche una classificazione delle indagini bibliografiche in cronologica, geografica, disciplinare, un po' come accade anche per la Storia della letteratura, che, a differenza della Bibliografia, vede però restringere il suo campo di interesse e di azione a certe precise tipologie testuali. 
Dalla Storia della Bibliografia si sviluppò infatti in particolare nel XVIII secolo la Historia literaria che successivamente si è consolidata nella Storia della Letteratura di cui abbiamo appena detto, mentre la Bibliografia si fece a poco a poco in senso metaforico l'immagine riflessa nello specchio della Storia della Cultura, da intendere come dello Zeitgeist, delle mappature, delle conoscenze e della stratificazione del sapere.

Se la biblioteca è il sistema che mette in rapporto questi elementi:

a) Autori (volontà intellettuali)

b) Libri (Opere + Edizioni: manifestazioni intellettuali e materiali)

c) Cataloghi (insieme di indici, scheletro delle mappe categoriali, mediatori)

d) Utenti (esigenze informazionali consapevoli, ricezioni inconsapevoli di offerte informazionali)

... E se la biblioteca è composta da questi elementi costitutivi:

a) Fondatore (imprinting)

b) Possessore/gestore (pubblica/privata - proprietà/accesso; statali, di enti, di comunità, di ordini, di associazioni, universitarie ...)

c) Raccolte (storica, moderna, di conservazione, di consultazione)

d) Scopi (mission)

e) Utenza

f) Strumenti (cataloghi - reali, per autori, per materie - e inventari)

g) Documenti (vari supporti)

... Allora nell'ambito della disciplina di Storia delle biblioteche si può ricostruire sia la storia di una istituzione che la storia del valore documentario e culturale di una collezione, quindi indirettamente la storia di "una cultura". In entrambi i casi, se si scende nello specifico, se ne descrive l'origine e la fondazione, la storia, la consistenza, la destinazione e l'uso, e la si contestualizza culturalmente. Ma nella Storia delle biblioteche ci sono difficoltà di non poco conto, che risiedono innanzitutto nel rapporto di grande indeterminatezza esistente tra gli elementi che abbiamo visto comporre una biblioteca. Tale distanza e siffatta rigidità aumentano nelle biblioteche di conservazione, nelle quali le stratificazioni librarie appartenenti ad epoche InCID: R. Ci. Inf. e Doc., Ribeirão Preto, v. 7, n. esp., p. 99-133, ago. 2016. 
e culture diverse fra loro e dall'attuale, sbilanciano totalmente il concetto di congruenza tra domanda ed offerta, in quanto diverse sono le mappe scientifiche e concettuali alle quali via via ci si riferisce e con le quali ci si confronta.

In ogni caso una "Storia della biblioteca" deve accompagnarsi sempre ad una "Storia delle biblioteche": accanto ad un'analisi evenemenziale (dei fatti che l'hanno vista protagonista) e ad una amministrativa, ne va infatti fatta una bibliografico-culturale (elemento che la distingue dalle altre istituzioni con compiti di carattere socio-assistenziale, come ad esempio le scuole e gli ospedali). È inoltre indispensabile muoversi poi nella Storia delle biblioteche cercando di individuare dei paradigmi, dei modelli di riferimento e di valore, a seconda della tipologia studiata, per poter realizzare dei processi di confronto. Nella pratica ciò significa mettersi nelle condizioni di poter misurare tanto il grado di copertura bibliografica (letteraria, scientifica, editoriale) che il livello delle logiche della sua organizzazione (cataloghi, classificazioni, disposizione fisica dei documenti).

D'altro canto le biblioteche rappresentano di fatto l'unica testimonianza della pubblicistica del passato e del presente, in quanto nessuna elaborazione bibliografica o storica oppure letteraria segnala, utilizza e tantomeno rispecchia tutto quello che c'è nelle biblioteche e che potrebbe venire utilizzato.

La Bibliografia è la disciplina che sottende a queste ricostruzioni storiche facendo emergere la humus bibliografica custodita nelle biblioteche, ma è anche quella che guida nella contemporaneità la formazione, la costruzione, e l'aggiornamento delle collezioni documentarie e degli strumenti atti a permetterne, diffonderne, e valorizzarne la conoscenza. Proprio su questi aspetti risiede la distinzione tra la Bibliografia e la Biblioteconomia che è la disciplina che si occupa specificatamente delle tecniche e delle tecnologie, quindi degli standard, delle norme e delle linee guida, ed ancora dei software e dei programmi che permettono alle biblioteche di qualificarsi come sistemi organizzati e gestionali. 


\section{Origini di una consapevolezza bibliografica e nascita della Bibliografia come disciplina}

La Bibliografia nasce, come è noto e come vedremo, con Conrad Gesner; tuttavia le sue origini possono essere rintracciate a partire da un millennio prima, ed è interessante seguire la maturazione di una consapevolezza bibliografica anche se di natura non ancora disciplinare quanto piuttosto strumentale e concreta.

Gli autori che hanno preceduto Johannes Trithemius e Conrad Gesner, accompagnando la Bibliografia nella sua concretizzazione disciplinare attraverso un iniziale De viris illustribus liber che si è man mano ampliato ed arricchito in un'ottica di "collaborazione naturale" (da oltre 130 voci fino a circa 300), sono: San Girolamo (IV sec. d.C.), Gennadio di Marsiglia (V sec.), Isidoro di Siviglia (VI sec.), Sigeberto di Gembloux (XI sec.), Onorio di Autun (XII sec.) e Enrico di Gent (XIII sec.).

Questi autori realizzano quella tipologia letteraria che va sotto il nome di "Prosopografia", con la quale si intende una composizione repertoriale il cui obiettivo non è ancora quello di realizzare uno strumento di reperimento efficiente ed efficace come può essere un promptuarium ma di comporre piuttosto una storia della letteratura tramite l'esposizione sintetica degli autori e delle loro opere. Per questo l'ordinamento interno delle voci degli autori è cronologico basato sulla loro comparsa operativo-letteraria nella storia. Lo stesso "indice", quando c'è, non fa che elencare quei nomi nello stesso ordine in cui si trovano nel testo principale del liber. Questa mentalità prosopografica sarà molto vicina alla stessa che moltissimi secoli dopo determinerà l'affermarsi di una tendenza historico-literaria col presupposto di ricostruire e dimostrare lo sviluppo e lo svolgimento della produzione scritta (in questa fase il riferimento della parola literaria è alla scrittura e non a una precisa sezione di produzione come la intendiamo oggi). Questo orientamento darà poi luogo a sua volta da un lato alla Storia della letteratura che appunto si concentrerà sui testi che oggi consideriamo base della nostra cultura scritta di prosa e poesia e dall'altro alla coltivazione di una bibliografia nazionale come rappresentanza storico-politica-geografica della produzione di scritti e della loro pubblicazione in un "ambito culturale confinato".

Di seguito alcune esemplificazioni tratte da una edizione incunabola del Liber de viris illustribus di San Girolamo (FIG. 1). Qui una pagina con numerose voci di autori, segnalate da una iniziale onciale evidenziata, con biografie stringate e la citazione delle opere scritte dai singoli autori:

InCID: R. Ci. Inf. e Doc., Ribeirão Preto, v. 7, n. esp., p. 99-133, ago. 2016. 
Natura ed origini della Bibliografia: una prospettiva disciplinare per la contemporaneità

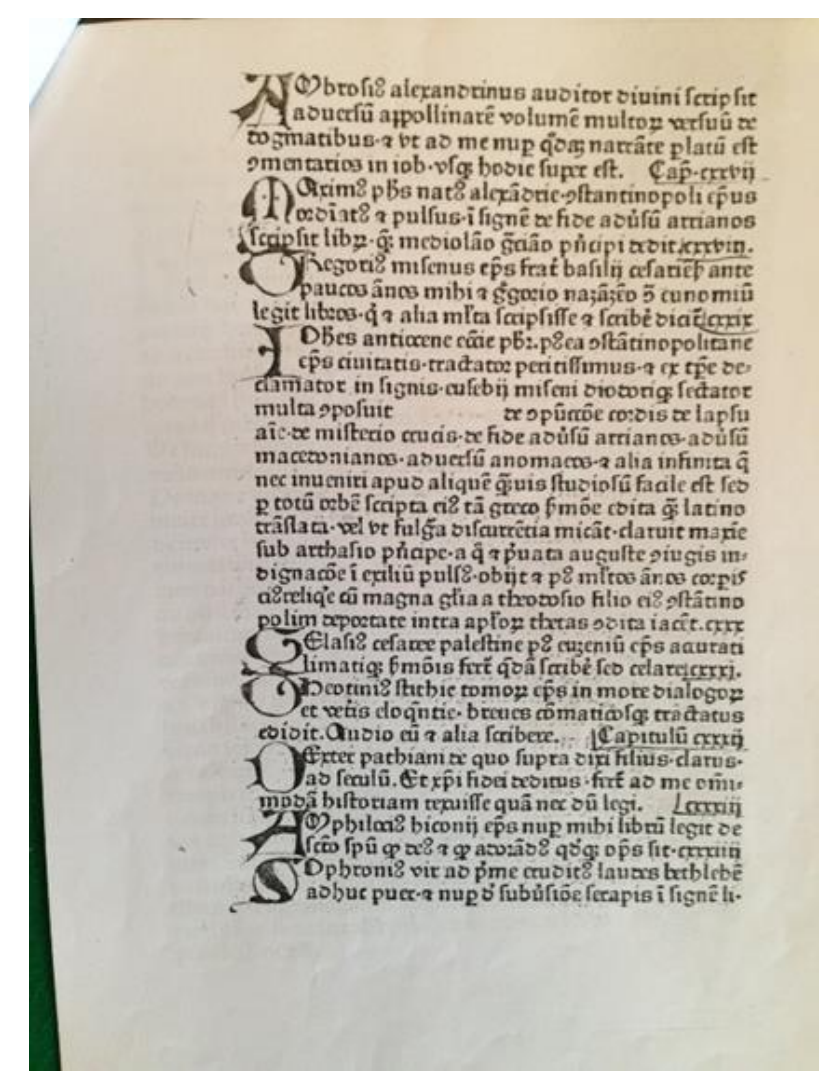

FIGURA 1 - Liber de viris illustribus - Voci di autori Augsburg: Günter Zainer, [ante 1470?]

Riproduzione: archivio privato di Alfredo Serrai

In questa immagine seguente (FIG. 2) vediamo invece l'indice degli autori nell'ordine della loro comparsa nel testo; si tratta più che di un indice di un 'sommario' che qui viene chiamato metaforicamente oculus ad indicare con il termine 'occhio' l'azione di rapida cattura visiva delle voci messe in evidenza. 


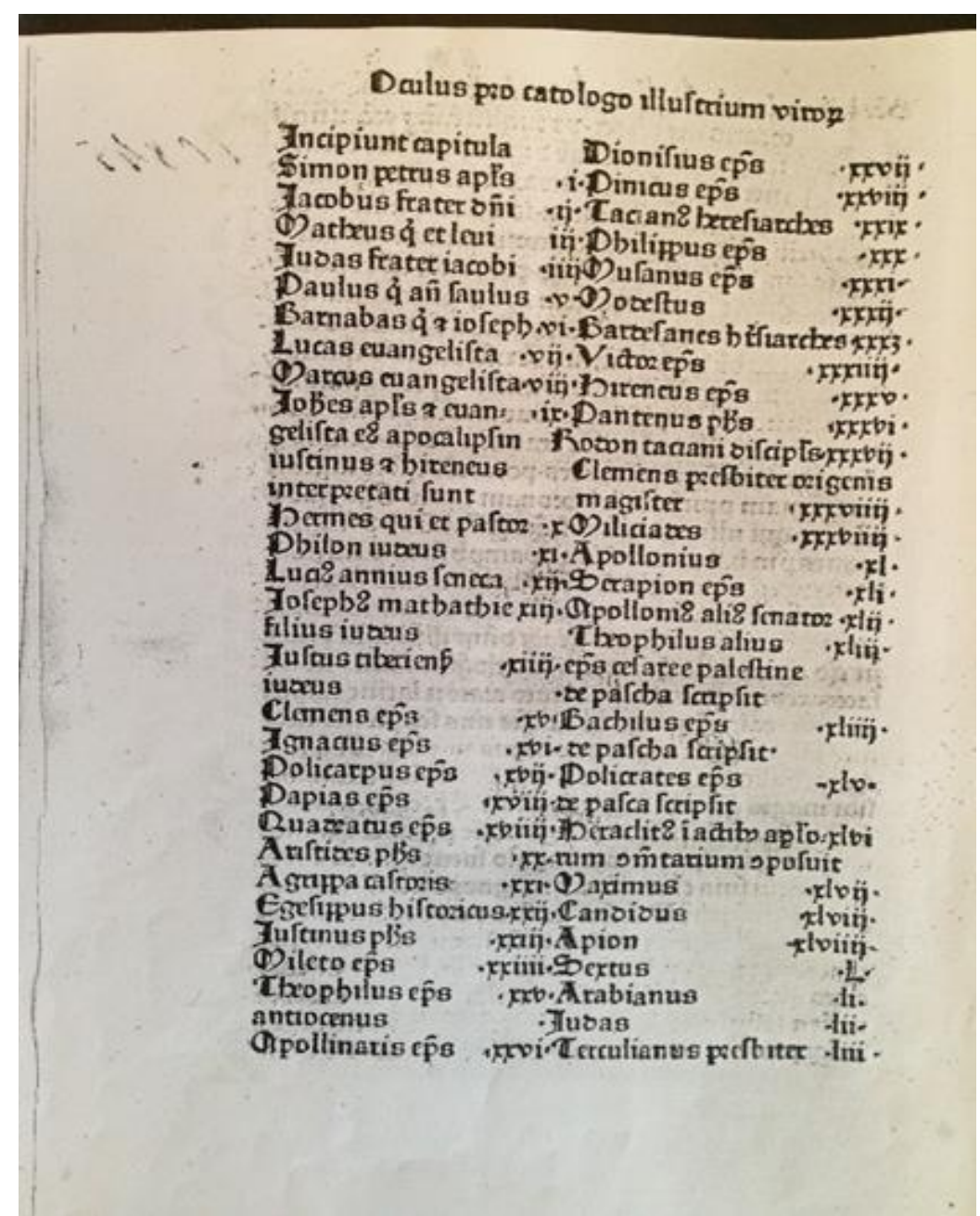

FIGURA 2 - Liber de viris illustribus - L'indice degli autori nell'ordine della loro comparsa nel testo Augsburg: Günter Zainer, [ante 1470?]

Riproduzione: archivio privato di Alfredo Serrai

Non si può infine non segnalare anche l'apparizione in questo liber di San Girolamo della prima "autobibliografia" (FIG. 3) alla quale vediamo l'autore dedica molto spazio! 


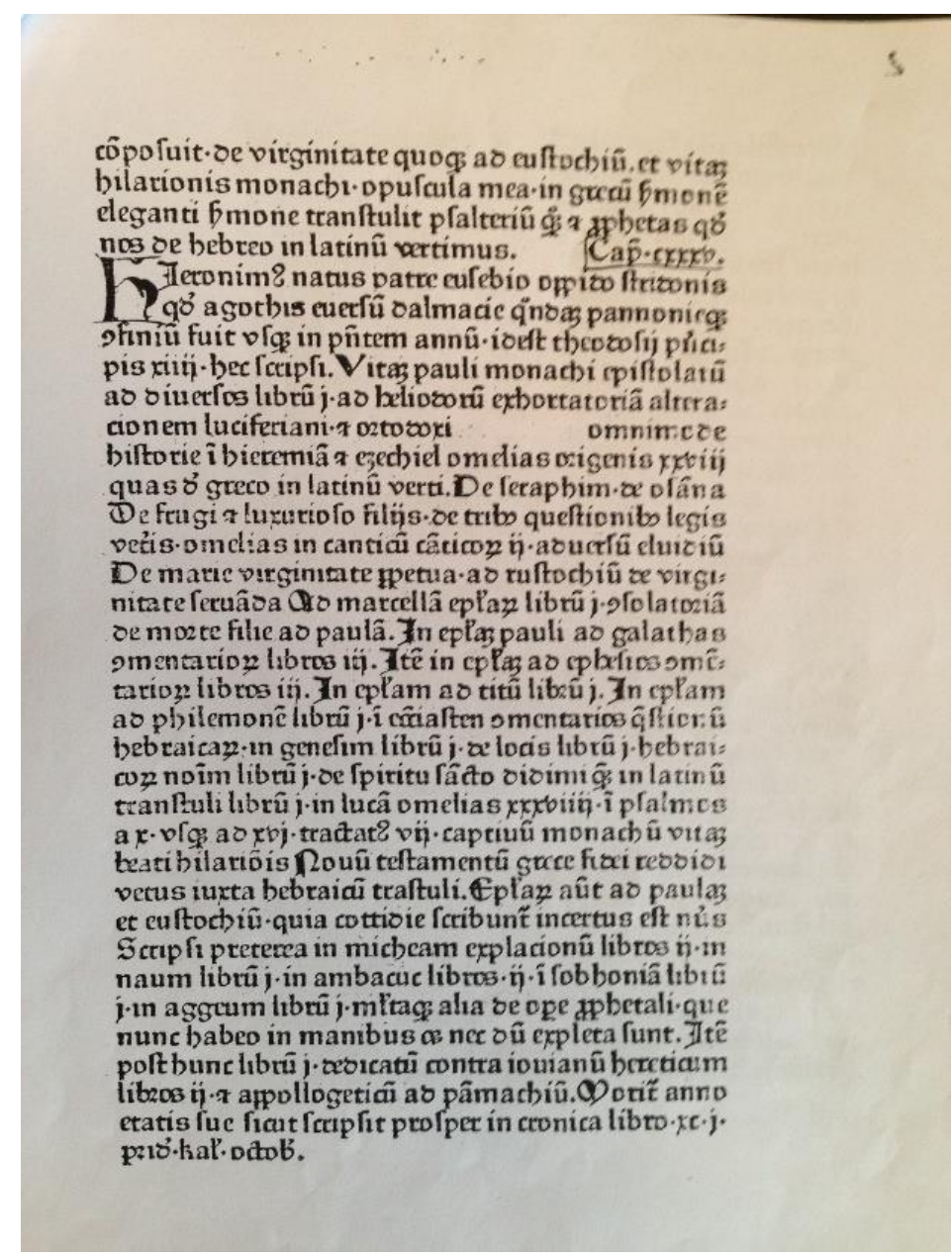

FIGURA 3 - Liber de viris illustribus - Autobibliografia Augsburg: Günter Zainer, [ante 1470?]

Riproduzione: archivio privato di Alfredo Serrai

Abbiamo citato gli indici di nomi, ma non meno importante e certamente più complessa è invece la questione della indicizzazione semantica. Insieme agli Indices scriptorum esistevano gli Indices verborum et rerum, ma non si trattava ancora dell'indice per 'soggetto' come concetto quanto di un indice per parole notevoli e rilevanti presenti nei testi.

La funzione di evidenza concettuale veniva invece espressa dai loci; in particolare vi era la pratica di comporre dei loci teologici, ma sarà solo Gesner a dare alla entità bibliografica dei loci una architettura semantica universale, pura e strutturata. E vedremo che Gesner sarà un anticipatore rispetto a soluzioni che oggi facciamo fatica a costruire, a causa soprattutto delle rigidità di natura linguistica mentre al contempo si aspira sempre più ad una visione internazionale, integrativa, di scambio e collaborazione.

La "millenaria catena bibliografica", come è chiamato l'arco di questi scrittori prosopografici, si concluderà con il benedettino tedesco Johannes Trithemius (Johannes Zeller, 
detto Trithemius dal luogo di nascita Trittenheim, nato nel 1462 e morto a Würzburg nel 1516) personaggio religioso ricordato oltre che per i suoi lavori storici, bibliografici e devozionali anche per lavori di crittografia come la sua Steganographia.

Trithemius pubblicò per la prima volta a stampa il suo importante Liber de scriptoribus ecclesiasticis (FIG. 4) a Basel per Johannes Amerbach nel 1494, dopo alcune stesure manoscritte tra il 1487 e il 1492. A questa seguirono poi altre tre edizioni simbolo del successo che l'opera ebbe: Paris, Berthold Rembolt, 1512 (FIG.5); Köln, Peter Quentell, una nel 1531 e una nel 1546.

Il repertorio contiene 963 biobibliografie esposte secondo un impianto tabellare derivato dall'eredità manoscritta (titolo dell'opera, numero dei libri e incipit). Si tratta di un enorme incremento di notizie rispetto all'ultimo repertorio che abbiamo considerato nella "catena" che è quello redatto da Enrico di Gent!

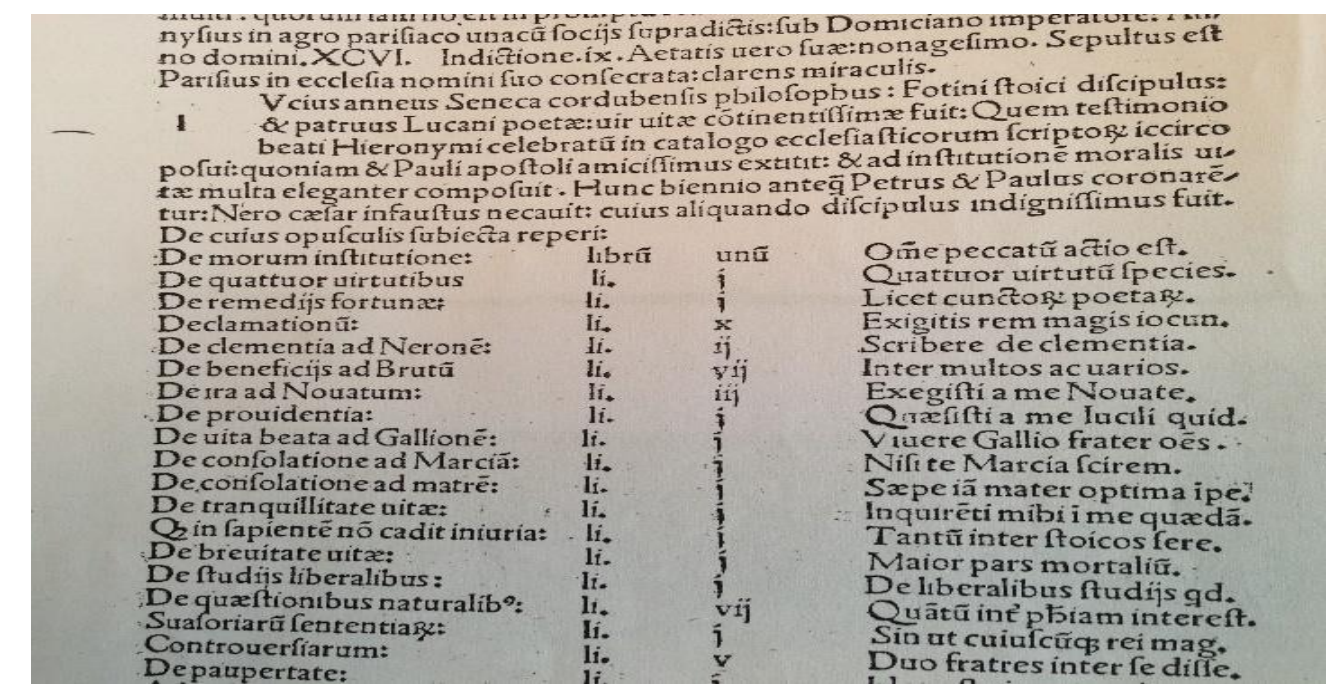

FIGURA 4 - Liber de scriptoribus ecclesiasticis - Impianto tabellare preso dall'edizione del 1494 Riproduzione: archivio privato di Alfredo Serrai 


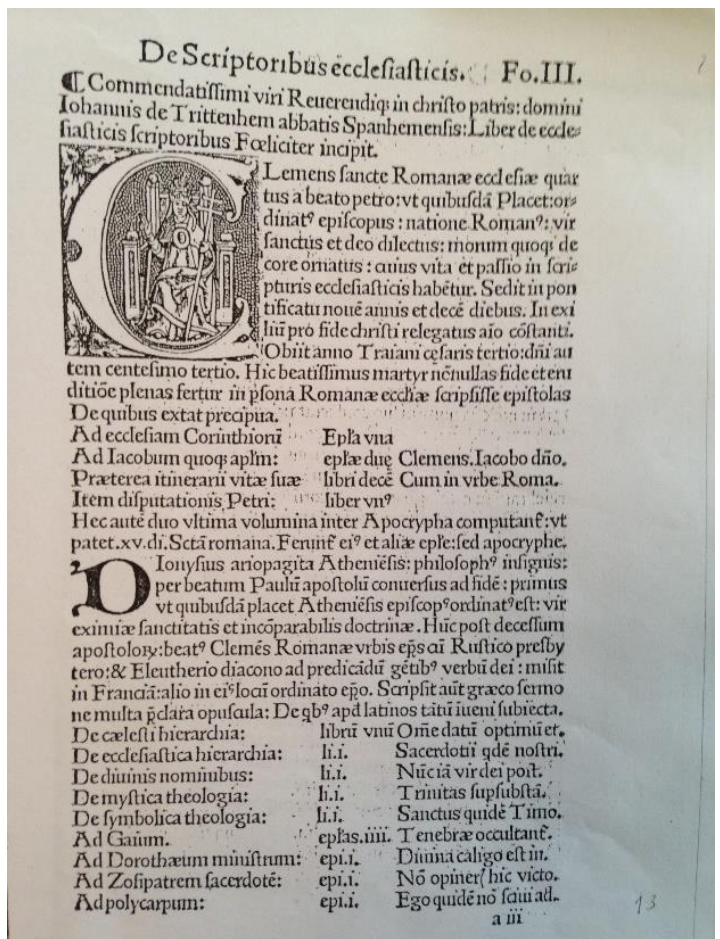

FIGURA 5 - Liber de scriptoribus ecclesiasticis - Esempio di biobibliografia tratta dall'edizione del 1512

Riproduzione: archivio privato di Alfredo Serrai

La novità indicale rappresentata dal repertorio bibliografico trithemiano sta nell'aver proposto un indice alfabetico finale dei nomi (FIG. 6) a vantaggio consultativo delle voci del testo principale ordinate sempre cronologicamente per periodo di massima fama (indicato col verbo "floruit"). Trithemius si qualifica dunque come una sorta di "nonno della Bibliografia" poiché testimonia la nascita di una consapevolezza indicale anche repertoriale e prontuaristica, e dunque bibliografica. La sua lucidità però non sta tanto nell'ordinamento in quanto alfabetico, ma in un ordinamento dell'indice differenziato rispetto a quello del testo, in modo che l'indice non sia più un sunto del contenuto ma sia uno strumento logico di reperimento e consultazione dell'opera. 


\section{ANNOTATIO SCRIPTORVM}

\section{$5 c \cdot n$}

Annotatio Scriptorum in bù so pere cómemoratorú:cơm appolitione numeri ordinem \& locû̉ uniufcuiufç eotum demonitrantis.

1. Cbacius epifcopus. Fol. 15

a Acurfius,ll. 64

Adamabbas. $\quad 52$

Adam wernberus. $\quad 139$

Adamãtius Origenes. 6

Adamánus abbas.

Adelmus ễus.

Adelboldus epus $\quad 39$

Adelmänus epus.

Adelpbusabbas.

Adilredus abbas.

Adrian 9 papa primus.

Adreualdus monacb?

Alanus de infulis.

Alanus de Rupe ... j2

Albertus abbas.

Albertus monacbus. $\quad 61$

Albertus patriarcba.
Albertus galioti.ll.

Albertus magn?.

Albertus Patauin? $\quad 77$

AlbertusBrixianus, 86

Albericus Roxiat. 11 . $4: 87$

Albertus de Eybe. $\quad 120$

Albertus de Prufa.

Alexander épus.

Alexíder papatertio 60

Alexander de Ales. $\quad 67$

AlexanderDolenfis. 67

Alexander de Alexádriar 78

Alexader de Alexander papa.v. . joo

Alexander papa.v. $\quad 122$

Alcuin' fue Albunus.

Albo Floriaceñ,abbasi

Algerus monacbus. … 50

Almānts monacb?

Alpbanus archiépus.

Alpbarus minor.

Alpbarus minor.

Alpbontus rex.

Alpbonfus epus.

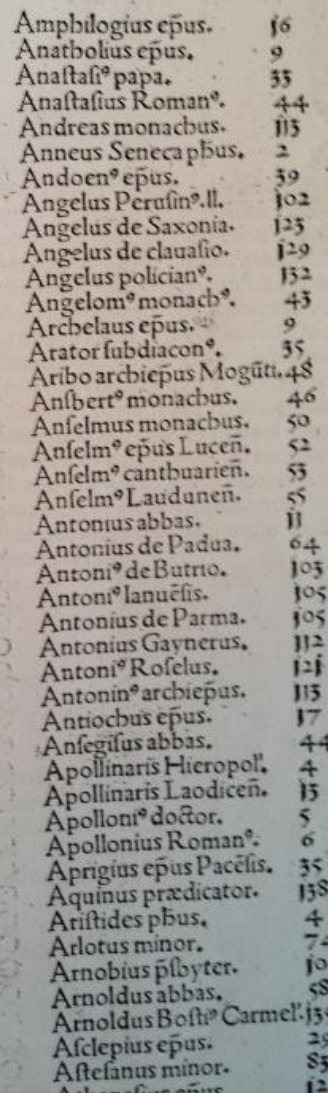

FIGURA 6 - Liber de scriptoribus ecclesiasticis - Indice finale alfabetico tratto dall'edizione del 1494 Riproduzione: archivio privato di Alfredo Serrai 


\section{Il progetto bibliografico di Gesner}

Se Trithemius è l'antenato della Bibliografia, il padre fondatore è Conrad Gesner (Zurigo, 1516-1565), scienziato, erudito e bibliografo zurighese. Si tratta di un personaggio ancora da conoscere a fondo, nonostante sia stato più volte studiato accuratamente in chiave tematica, dalle sue opere botaniche alla sua biblioteca personale alla sua visione teologica. Non sempre si è trattato di trattazioni scientifiche, in alcuni casi Gesner è stato superficialmente citato utilizzando il riconoscimento che gli deriva dalla sua ecletticità di interessi di filologo, botanico, zoologo, medico, teologo, bibliografo...

A questo su profilo enciclopedico, ma tuttavia compatto, contribuì la vicinanza con personalità religiose di spicco nella Riforma protestante, prima fra tutte quella di Ulrich Zwingli (morto nel 1531) che nella sua visione teologica - che dette luogo alla particolare corrente "zwingliana" distintasi dal luteranesimo e dal calvinismo - riuniva ed armonizzava i principi della teologia cristiana, con l'eredità intellettuale della civiltà classica e pagana, e addirittura con l'esercizio della ragione umana. Zwingli fu davvero un uomo del suo tempo, un pensatore rinascimentale, per il quale Dio corrispondeva a verità e bene supremi, distribuendo a tutti la possibilità di accedere al vero e di potersi pertanto salvare fin dal momento della creazione e non solo con l'incarnazione come storicamente dogmatizzato. La tradizione pagana e la teologia cristiana venivano così a trovarsi comprese nello stesso quadro filosofico e culturale. Ciò legittimò pertanto Gesner, che ne era seguace, a concretizzare un'opera bibliografica che comprendesse indistintamente tutti coloro che avevano fino ad allora scritto opere degne di memoria.

A noi interessa infatti proprio la monumentale opera bibliografica di Gesner, dove si trovano impegnati, sintetizzati e rappresentati iconograficamente tutti i suoi interessi, le sue visioni, e le sue esperienze in una struttura olistica, e dove ogni brandello di scibile ha potuto trovare il posto spettantegli nell'universo del sapere.

Gesner dette alla luce delle stampe la Bibliotheca Universalis (FIG. 7) edita a Zürich dal tipografo Christoph Froschauer nel 1545, cui seguirono le Pandectae pubblicate in due volumi, il primo nel 1548 (FIG. 8) e il secondo nel 1549 (FIG. 9). I tre volumi vengono dati alle stampe da Gesner separatamente, tuttavia - come ho potuto dimostrare - si integrano perfettamente in un unico progetto bibliografico. Si tratta infatti di un immenso lavoro bibliografico caratterizzato dall'intenzione di offrire il quadro totale della realtà scientificoletteraria della metà del XVI secolo. 


\section{BIBLIOTHECA}

Vniuerfalis, fiue Catalogusomnis um faiptorum locupletiffimus, in tribus linguis, Latina, Grace, $8<\mathrm{He}$ braica: extantium $\&$ non extantiü, ueterum \& recentiorum in hunc uf $q$ diem, doctorum $\&$ indoctorum, publicatorum \& in Bibliothecis laten= tum. Opus nouum, \& nō Bibliothecis tantum publicis priuatisue in=

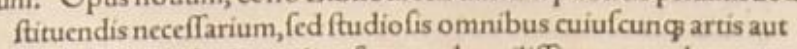

feientix adftudia melius formanda utilifimum : authore conRADo gesnero Tigurino doctore medico.

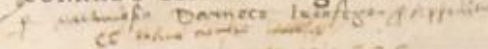

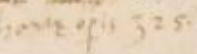

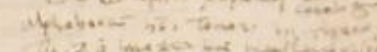

FIGURA 7 - Titolo tratto dal frontespizio della Bibliotheca Universalis (1545)

Riproduzione: archivio privato di Alfredo Serrai

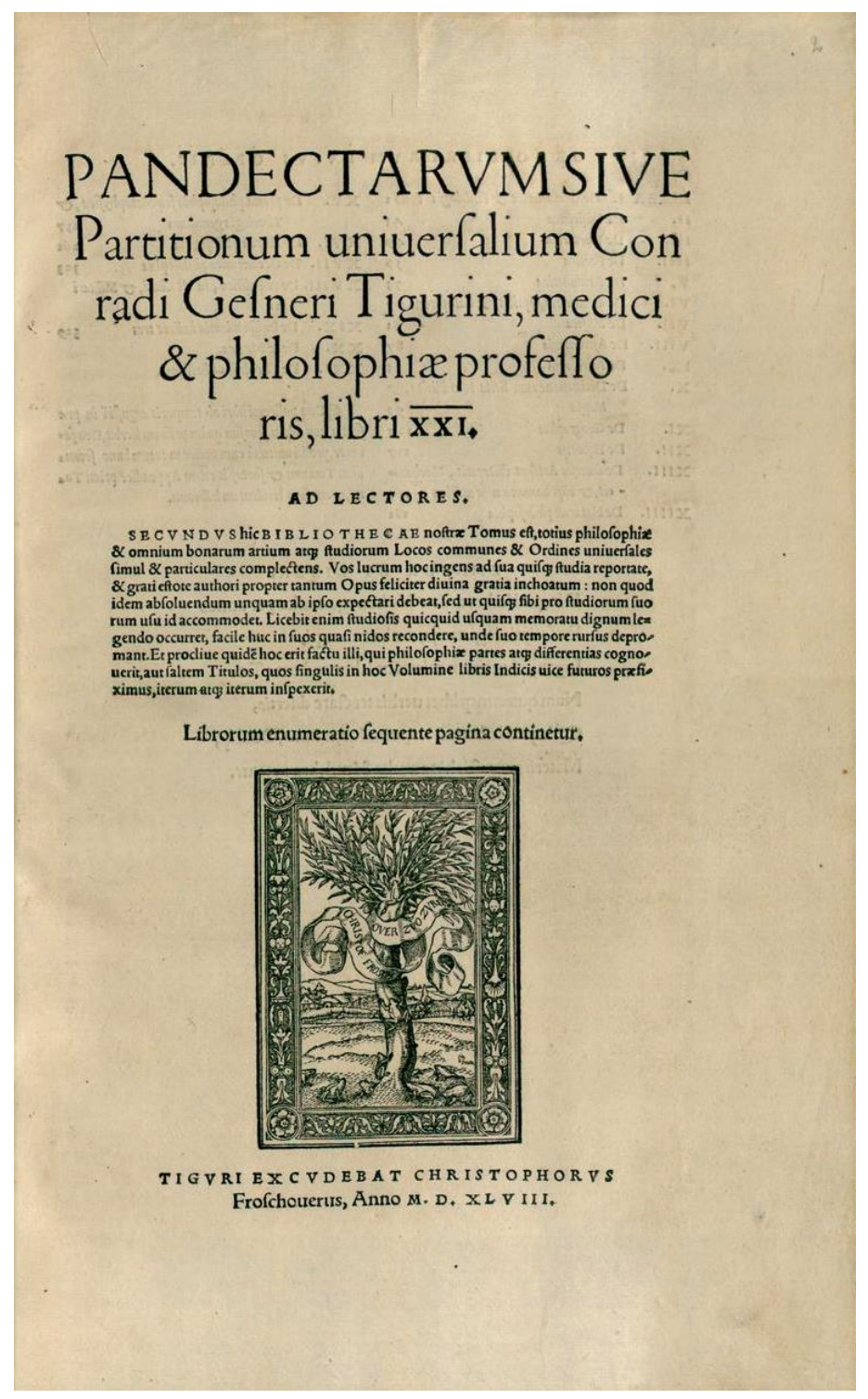

FIGURA 8 - Frontespizio del I volume delle Pandectae (1548) Disponíbile: <http://www.e-rara.ch/zuz/content/pageview/1160095>

InCID: R. Ci. Inf. e Doc., Ribeirão Preto, v. 7, n. esp., p. 99-133, ago. 2016. 


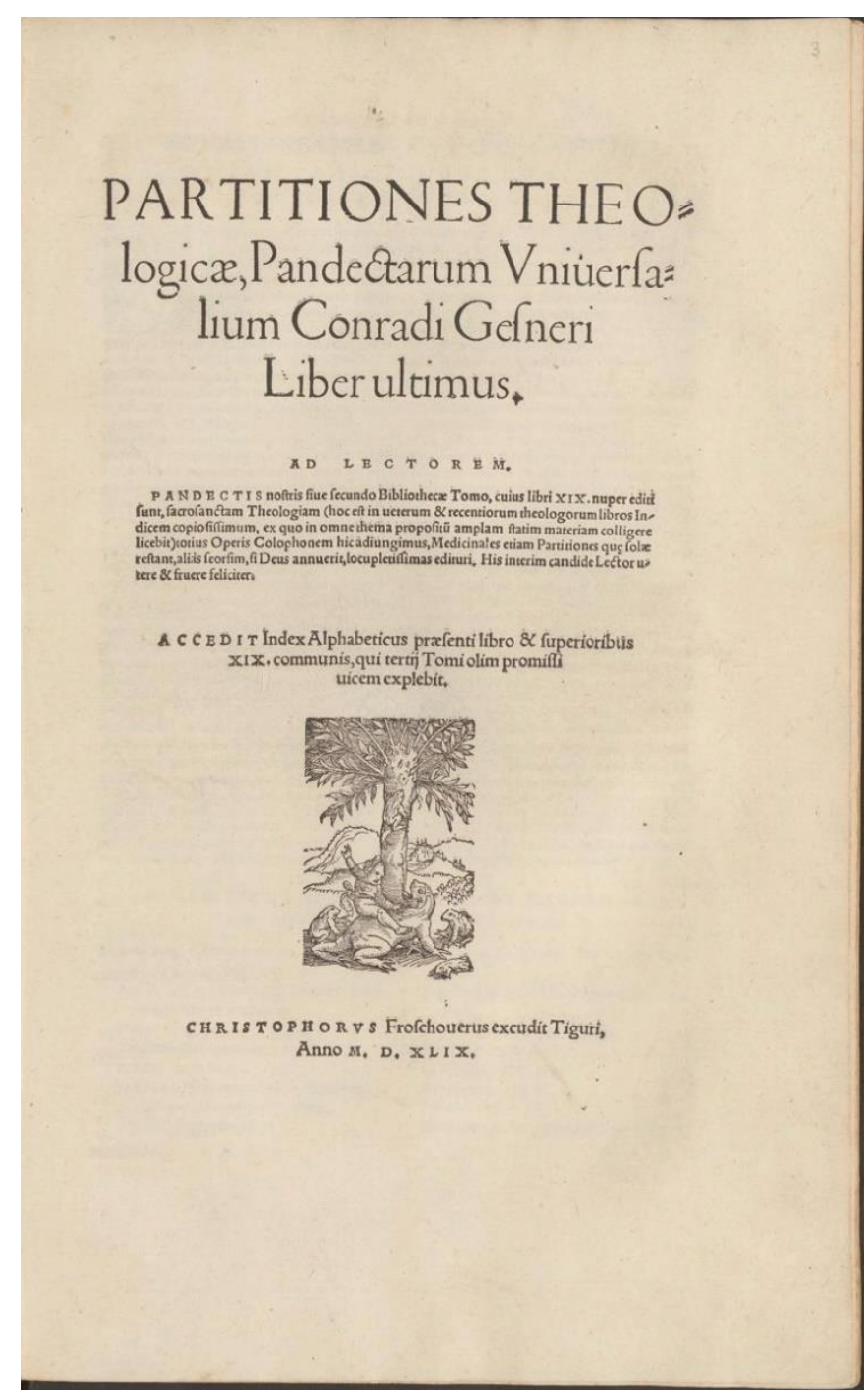

FIGURA 9 - Frontespizio del II volume delle Pandectae (1549)

Disponíbile: <http://www.e-rara.ch/zuz/content/pageview/678541>

L'unica chiave per comprendere l'effettiva portata informativa del repertorio, sia in termini documentari che esegetici, è stata quella di considerare le fonti di informazione utilizzate per comporlo e la sua rappresentatività bibliografica rispetto alla cultura scritta reperibile in quell'epoca. Conseguentemente è stato possibile misurarne il grado di 'universalità' rispetto ai propositi desiderati e agli strumenti disponibili. Questo non era stato sviscerato, appunto, prima della pubblicazione del mio volume La Bibliotheca Universalis di Conrad Gesner: monumento della cultura europea (Roma, Bulzoni, 2012), nel quale è confluita anche la mia tesi di Dottorato di ricerca in Scienze bibliografiche, archivistiche e documentarie discussa ad Udine nel 2009. Il lavoro è però uscito alle stampe tre anni dopo, in quanto la mia analisi sul repertorio era continuata ed è stato quindi necessario rielaborare i risultati al fine di poterli divulgare in una forma comprensibile anche ai non esperti di Bibliografia. Era ormai un dato di fatto appunto che l'interesse per Conrad Gesner, e di conseguenza la sua notorietà, si 
stavano espandendo rapidamente e "popolarizzando" anche in vista delle sue prossime celebrazioni previste per il 2016.

\section{Le fonti della 'Bibliotheca Universalis'}

Le fonti da cui sarebbe stato attinto il materiale si trovano segnalate nella Epistola nuncupatoria della Bibliotheca, e in alcune parti dei Pandectae, ma spesso anche nel testo vero e proprio del repertorio. In generale le segnalazioni bibliografiche, sia quelle soltanto letterarie che quelle editoriali, sono derivate da:

- le autopsie effettuate personalmente su materiale librario posseduto da Gesner stesso, e dalla sua cerchia di amici ed eruditi, in particolare medici e filologi, oppure conservato in biblioteche cui egli aveva avuto accesso nel corso dei suoi numerosissimi viaggi;

- la corrispondenza con gli amici e le conversazioni avute con i dotti;

- i cataloghi dei tipografi e dei librai reperiti alle fiere nundinarie o che gli erano stati inviati;

- gli indici delle biblioteche pubbliche e private, sia ispezionati di persona che ottenuti in copia;

- le repertoriazioni bibliografiche che avessero preceduto la sua intendendovi sia delle opere con contenuto bibliografico, che veri e propri repertori bibliografici; Gesner ha utilizzato infatti una gran varietà di repertori, da quelli dei prosopografi che lo avevano preceduto - quindi da San Girolamo fino all'abate Johannes Trithemius - a quelli di specialisti di Diritto, Poesia, Medicina e Botanica (Lilio Gregorio Giraldi, Pietro Crinito, Symphorien Champier, Otho Brunfels, Leonhard Fuchs, Raffele Maffei detto Volaterranus, Johann Fichard, Bernardino Rutilio, Giovanni Nevizzano...).

La parte più cospicua dei "recipienti informativi" da cui Gesner ricavò notizie deriva però innegabilmente da suoi viaggi, primo fra tutti quello in Italia del 1543. Gesner si era recato in quel periodo a Ferrara, Verona, Como, Bologna e Venezia, invitato in quest'ultima dall'ambasciatore spagnolo Diego Hurtado de Mendoza.

La collaborazione fra studiosi ed eruditi era la base della comunità letteraria, lo scambio di testi e notizie era un bene comune. Vi erano occasioni annuali di incontro, come i soggiorni 
alle terme o le fiere delle Nundine, dalle quali nascevano collaborazioni, amicizie, inviti reciproci, e tutto ciò facilitava insieme alla circolazione degli eruditi, anche e ma soprattutto quella delle notizie di testi e di libri, nonché di libri stessi e anche di copie dei cataloghi. Le città italiane, divenute le istituzioni-asilo del patrimonio librario greco in seguito alla fuga dei greci da Corfù, erano diventate una meta ambita e privilegiata, in particolare per questo genere di letteratura erano visitate, frequentate e scelte per studiarvi anzitutto le città di Venezia e Padova.

\section{L'importanza e il ruolo dei repertori bibliografici nella tradizione culturale}

La tradizione precedente andava conosciuta, molta ancora scoperta, per poi venir utilizzata quale base di nuove ricerche e di nuove imprese di studio e divulgazione. Non si erano affermati ancora i concetti di plagio, di diritto d'autore, di paternità autoriale sottratta, ma gli uomini di lettere erano generalmente mossi nell'ambito di una ecumenicità straordinaria. Non si trattava di scoprire strade sconosciute, ma di seguire perfezionando, approfondendo e documentando quanto la civiltà umana aveva via via tracciato, al fin di darne sempre esplicito pubblico conto.

Ma, oltre a fissare i connotati del patrimonio scientifico e letterario, i repertori finivano per fotografare e fissare lo status di singole collezioni e di biblioteche, nonché di epoche e delle loro tendenze e preferenze culturali. Vi sono compresi ambienti religiosi e laici, biblioteche pubbliche e biblioteche private, quest'ultime non di rado per liberalità di accesso e consultazione più pubbliche di quelle che lo erano per gestione e definizione statutaria. Paradossalmente proprio per questo motivo quelle personali erano le raccolte più ambite dagli eruditi, le più ricche e quelle più vicine alla perfezione dal punto di vista bibliografico.

Dall'analisi di una qualsiasi delle collezioni private tra le tante citate nella Bibliotheca emerge come, grazie al repertorio gesneriano, sia possibile ricostruire, pur se a volte parzialmente, la collezione libraria di un erudito, sia nella sua composizione che nella sua qualità letteraria e bibliografica, e come, quindi, il repertorio gesneriano si confermi in tal modo proprio nella funzione di testimonianza, spesso unica, relativamente a raccolte librarie private interessanti ai fini della ricostruzione dell'ambiente erudito del XVI secolo.

Gesner, anche quando nella prefazione alla Bibliotheca parla della necessità di registrare il patrimonio per garantire ad esso almeno notizia nel futuro, non aveva in mente di realizzare 
una testimonianza scritta di singole biblioteche o raccolte, tuttavia questo è avvenuto poi involontariamente rispetto agli intenti primari. Egli infatti non si era tanto concentrato sugli esemplari e sulle loro caratteristiche, e nemmeno sulle edizioni, ma - e questo è il fatto bibliografico principale - sulle opere e sui testi. Gesner non ha realizzato un repertorio "universale" completo di tutte le edizioni manoscritte e a stampa raggiunte, ma di tutte le opere di cui aveva avuto notizia sia diretta che indiretta, anche se si trattava di opere andate perdute. Quando inoltre, da filologo esperto, egli forniva la segnalazione di più esemplari da collazionare, lo faceva con uno scopo preciso, ossia per dare quella documentazione che poteva servire per produrre nuove edizioni a stampa più corrette delle precedenti. Il focus era dunque quindi il miglioramento della produzione intellettuale, letteraria e scientifica.

A costituire il tessuto base della Bibliotheca sono state quindi le fonti catalografiche, quelle di tipo letterario-bibliografico inerenti in particolare il Diritto, la Medicina, la Poesia, e le bibliografie vere e proprie, ossia nella terminologia gesneriana i Catalogi scriptorum.

Ricchissimo serbatoio informativo erano stati alcuni cataloghi di biblioteche in particolare italiane: a Venezia quella del Bessarione, quella di Hurtado de Mendoza, di Sant'Antonio del Castello, del Monastero di San Giovanni e Paolo; a Bologna la Biblioteca di San Salvatore; mentre a Roma la Vaticana, e a Firenze la Mediceo-Laurenziana. Ma si trova registrata anche una notevole presenza di raccolte straniere come quelle private di Desiderius Erasmus, di Conrad Peutinger, di Cristoph Clauser, di Alexander Brassicanus, oltre a quella quella pubblica di Augsburg.

Tra le fonti protagoniste della Bibliotheca abbiamo ricordato anche i cataloghi di vendita di tipografi ed editori. Particolare ricchezza è quella delle notizie bibliografiche provenienti dalle aree tedesca e francese, che sono quelle cui Gesner era maggiormente legato, seguite dall'area italiana, la cui presenza è giustificata dal fatto che vi si conservava la gran parte delle opere ancora inedite in lingua latina e soprattutto in quella greca.

Nel mio volume ho qualificato i cataloghi di editori, tipografi e librai come una "fonte occulta", in quanto non dichiarata apertamente da Gesner nelle declaratorie della Bibliotheca. A questa tipologia di fonti appartengono i cataloghi di vendita di alcuni editori pubblicati in introduzione a 7 dei 20 libri di cui le Pandectae gesnerianae si compongono, e sono quelli di Froschauer, Gymnich, Wechel, Gryphe, Frellon, Manuzio e Froben. 
Natura ed origini della Bibliografia: una prospettiva disciplinare per la contemporaneità

Si percepisce appieno da questa 'carrellata esegetica' come Gesner abbia lavorato incessantemente alla caccia di notizie su materiale letterario e bibliografico, utilizzando i canali più disparati. Ciò gli fu possibile nonostante le difficoltà comunicative e di spostamento, in quanto era supportato da una tradizione precedente molto robusta e da una società colta ed erudita, cosciente della preziosità di ciò che conosceva, maneggiava, possedeva e studiava, e che pertanto era collaborativa, generosa, impregnata di valori intellettuali alti e nobili. La collaborazione fra gli studiosi era alla base della realizzazione delle imprese bibliografiche di largo respiro; ognuno metteva a disposizione i testi e le notizie di cui era in possesso, vi erano dei veri e propri scambi, e tutto ciò che ne derivava veniva concepito innanzitutto come bene comune, e poi soltanto indirettamente come merito personale.

I repertori bibliografici svolgevano come detto, per forza di cose, anche le funzioni non solo di mera registrazione del patrimonio scritto, ma di attestazione ed evidenza di quanto non fosse ancora stato impresso, e soprattutto di pubblicizzazione dello stesso perché se ne conoscesse l'esistenza e se ne promuovesse l'edizione a stampa. Ma oltre a fissare i connotati del patrimonio scientifico e letterario, i repertori finivano indirettamente per fotografare lo status di singole istituzioni librarie, o di semplici collezioni, e di conseguenza la humus culturale delle città in cui queste si trovavano. Molte di queste raccolte, come abbiamo già segnalato, pur non essendo ancora propriamente cittadine, ma private come origine e come mantenimento, erano tuttavia "pubbliche", laddove il concetto di pubblico non si riferiva alla proprietà ma al libero e consentito accesso agli studiosi. 


\section{L'indagine semantica gesneriana nelle Pandectae}

Ma veniamo alle Pandectae che rappresentano la seconda parte del progetto bibliografico gesneriano, e di fatto la sezione più complessa. Per la loro comprensione si è obbligati ad un riferimento continuo alla prima parte dove le informazioni sono state registrate da Gesner in una forma estesa e non sintetica e quasi 'siglata' come in questa seconda. Esse si rivelano in tutta la loro utilità e preziosità informativa solo se, dunque, si conosce bene il contenuto, l'architettura e il concreto apporto bibliografico della prima parte. La difficoltà di interpretazione delle Pandectae ne ha ostacolato la diffusione commerciale editoriale e di conseguenza lo studio e la ricerca; ma adesso che si sono invece diffuse le copie digitali a pubblico accesso on line della sua edizione (1548 e 1549), si riscontra un'esplosione del loro impiego ai fini ricostruttivi della storia bibliografica delle singole discipline: in particolare per ora della la musica, della medicina, e delle arti applicate.

Le Pandectae sono composte da 21 libri, i primi 19 pubblicati nel I volume del 1548 e il XXI nel volume del 1549. Resta fuori il XX dedicato alla Medicina che non vide la luce in tempo, come se Gesner dedicasse una cura particolare a quello che rispecchiava la sua formazione professionale principale. Della preparazione del XX libro abbiamo però testimonianza attraverso il materiale bibliografico medico raccolto da Hieronymus Wolph di cui oggi restano presso la Zentralbibliothek zurighese tre volumi manoscritti da questi curati nel 1596, mentre una parte del materiale risulta arrivata in Norvegia per passaggi di eredità e di vendita. Il progetto delle Pandectae portato quindi quasi completamente a termine è composto dai Libri:

I. De grammatica et philologia

II. De dialectica

III. De rhetorica

IV. De poetica

V. De arithmetica

VI. De geometria

VII. De musica

VIII. De astronomia

InCID: R. Ci. Inf. e Doc., Ribeirão Preto, v. 7, n. esp., p. 99-133, ago. 2016. 
Natura ed origini della Bibliografia: una prospettiva disciplinare per la contemporaneità

IX. De astrologia

$X$. De divinatione cum licita tum illicita et magia

XI. De geographia

XII. De historiis

XIII. De diversis artibus illiteratis, mechanicis et aliis humanae vitae utilibus

XIV. De naturali philosophia

$\mathrm{XV}$. De prima philosophia seu metaphysica et theologia gentilium

XVI. De morali philosophia

XVII. De oeconomica philosophia

XVIII. De re politica, id est civili et militari

XIX. De iurisprudentia indices tres

XX. De re medica

XXI. De theologia christiana

Interessante è anche analizzare la tavola delle classi che Gesner inserisce nell'ultimo volume e ultimo libro dedicato alla Teologia (FIG. 10), nella quale espone la sua personale visione culturale, che rispecchia probabilmente quella del suo ambiente, e ci fa meglio comprendere le difficoltà storico-ricostruttive che abbiamo precedentemente illustrato nella parte dedicata al rapporto tra la Bibliografia e la Storia delle biblioteche. Dalla Filosofia che sovrintende a tutte le arti e le scienze, si innestano discipline 'preparatorie' e discipline 'sostanziali'. Le preparatorie si specificano in 'necessarie' come quelle matematiche aritmetica, geometria, musica, astronomia e astrologia - e come quelle 'sermocinali' ossia per esporre - grammatica, retorica, poesia, e dialettica -, o 'ornanti' nel senso di utili a perfezionare ogni altra preparazione, come geografia, storia, magia e altre arti. Le 'sostanziali' invece sono costituite da fisica, metafisica, teologia cristiana, e etica nella quale sono comprese economia e politica che ha in se a sua volta la giurisprudenza. 


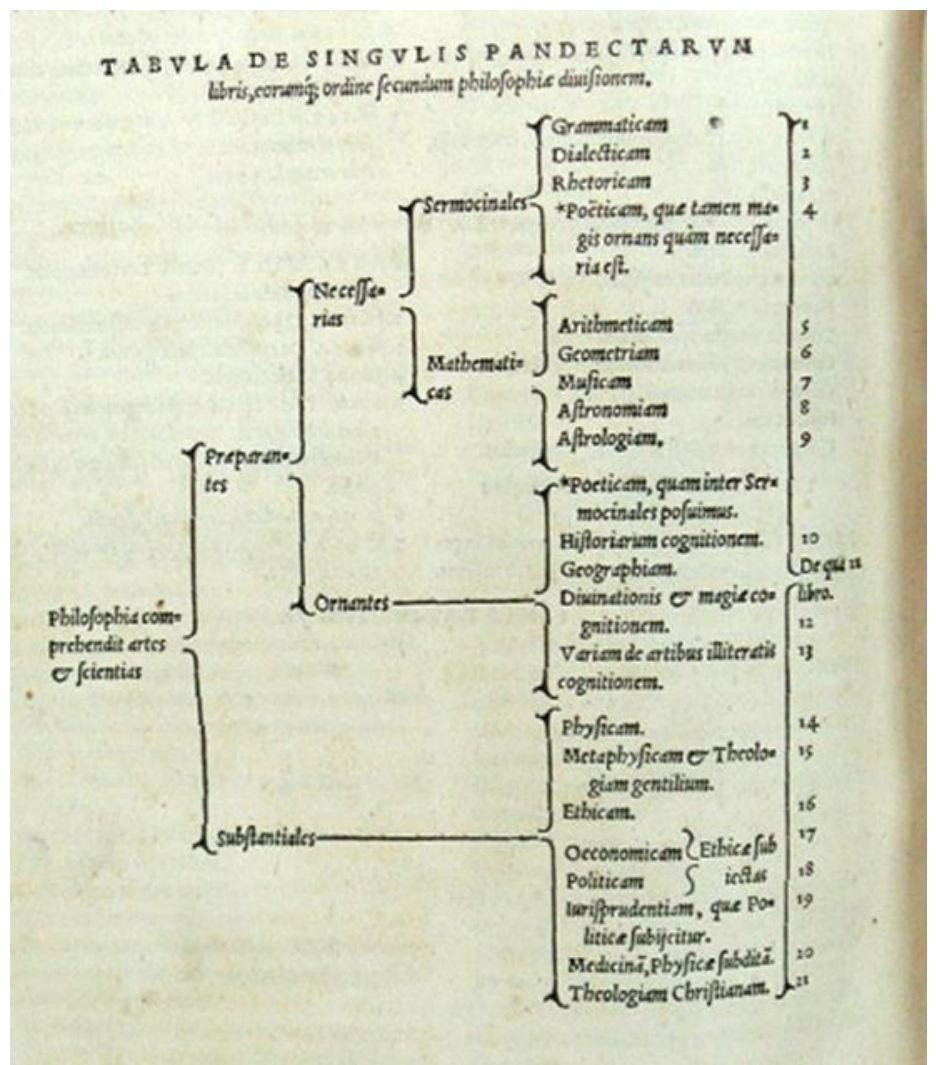

FIGURA 10 - Tabula delle classi tratta dal volume II delle Pandectae (1549)

Disponíbile: < http://www.e-rara.ch/zuz/content/pageview/625744>

I 21 (ma 20) libri contengono il materiale bibliografico già presente nella Bibliotheca Universalis sotto il nome degli autori, qui invece esposto mediante la granularizzazione del suo contenuto a partire dalla disciplina di riferimento fino ad arrivare al nucleo semantico chiamato locus communis o particularis, corrispondente grosso modo al nostro odierno soggetto catalografico. In più rispetto al volume del 1545 vi sono comprese le opere poligrafiche (es. di autori greci e latini con liste di loci communes), le opere prima non note, quelle anonime che nel volume del 1545 non potevano essere presentate sotto alcun autore, quelle ovviamente pubblicate dopo il 1545 o prima non note o conosciute da Gesner, ma anche gli scolia, i paragrafi, gli indici, le traduzioni, ed i compendi che nella struttura alfabetica non trovavano sufficiente 'autonomia' autoriale.

La architettura semantica vedeva quindi così ulteriori suddivisioni all'interno dei libri fino a giungere al nucleo semantico specifico:

Libri / Partitiones

Tituli

Partes

InCID: R. Ci. Inf. e Doc., Ribeirão Preto, v. 7, n. esp., p. 99-133, ago. 2016. 


\section{Paragraphi}

Loci communes

Loci particulares

Le Pandectae danno un apporto di rilievo alla storia delle classificazioni: la matrice semantica riunisce attraverso l'argomento tutte le presenze e i derivati testuali dell'opera (traduzioni, commentarii, epitomi, prefazioni...) ricostituendo così la configurazione completa di partecipazioni e contribuzioni che formano la progenie collaterale e successiva delle opere e delle edizioni.

Inoltre va sottolineato il fatto che quella applicata da Gesner ai Libri Pandectarum è una classificazione per concetti, per cui la stessa cosa viene classificata in modo diverso a seconda della disciplina in cui si trova; mentre nella III parte della Bibliotheca Universalis, quella inedita che doveva riguardare un indice alfabetico per soggetti, molte accezioni sarebbero invece scomparse come entità e come concetti. Si tratta questo di un punto cruciale per le classificazioni: mentre nei 21 libri gesneriani i concetti occupavano il posto che spettava loro nella architettura sistematica, la soggettazione moderna risulta a volte ancora inadeguata, ancora troppo ancorata all'impostazione di natura terminologica e linguistica piuttosto che a quella semantica, e proprio per ovviare a queste criticità sono state individuate nei tesauri e soggettari catalografici delle "faccette" per qualificare e definire le relazioni semantiche tra i termini (ma appunto non tra i concetti!). In tempi di scambi internazionali continui e di apertura immediata e globale dei prodotti scientifici è inaccettabile essere sottoposti ancora a tali vincoli linguistici, ma bisogna pur certo ammettere che Gesner aveva avuto la fortuna di potersi servire del latino che rappresentando una sorta di 'lingua franca' facilitava le operazioni rendendole comuni.

Per esemplificare le questioni su esposte si porta un esempio tratto dal Nuovo Soggettario italiano realizzato a cura della Biblioteca Nazionale Centrale di Firenze (FIG.11). 
Fiammetta Sabba

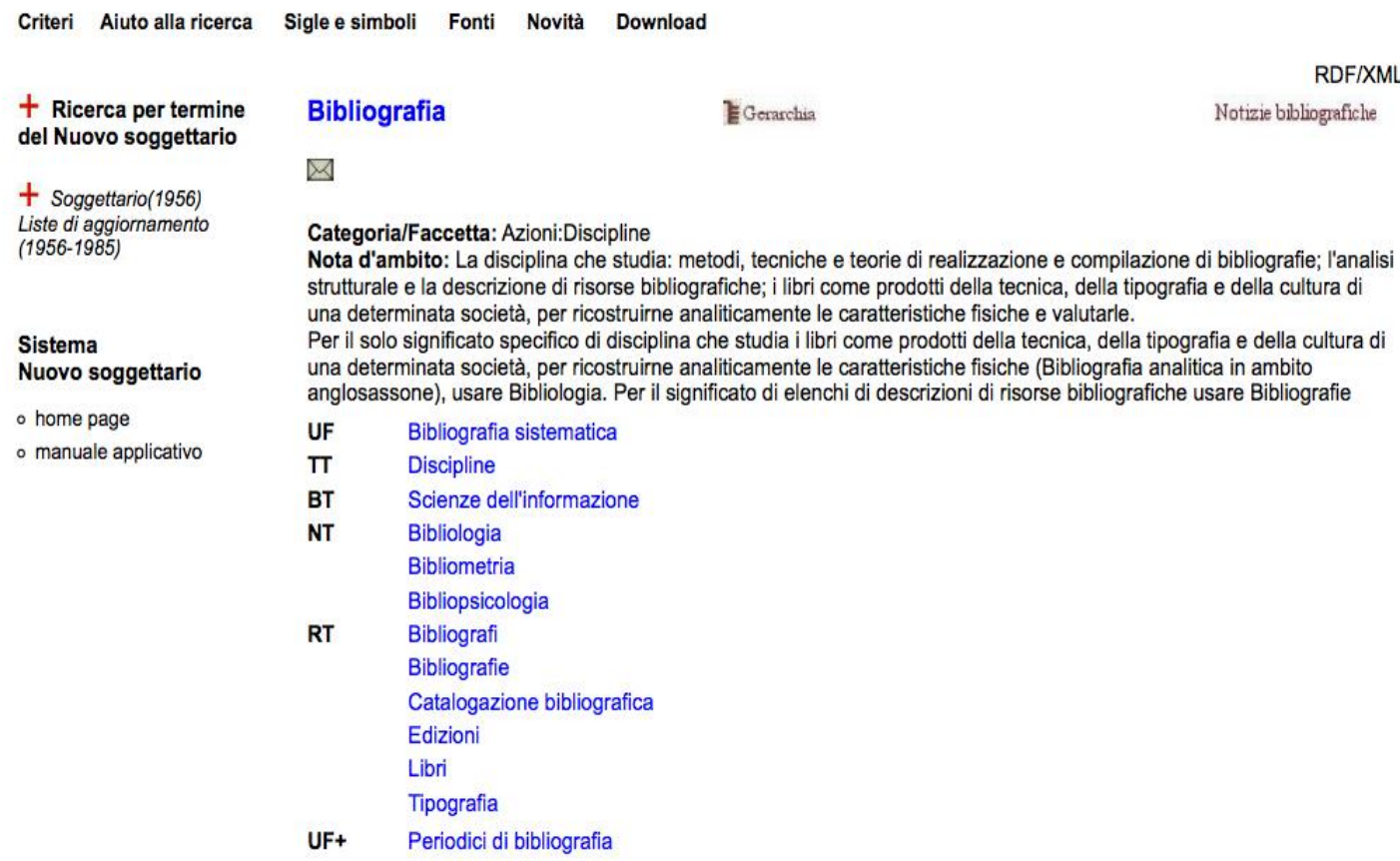

Nota storica: Precedentemente anche all'interno del termine composto: Periodici di bibliografia (BNI 1986-1998) Fonte: Soggetlario; PT; GBV; GBD; TRT; Wikipedia(IT) DDC (WebDewey): 010

Equiv. in altri strumenti di indicizzazione

LCSH: Bibliography

[1RAMEAU: Bibliographie

Agenzia catalografica/Proponente: BNI

Status del record: Termine strutturato

Identificativo: 5401

FIGURA 11 - Ricerca effettuata per 'Bibliografia' nel Thesaurus del Nuovo Soggettario italiano (Firenze, BNCF, 2007)

Disponíbile: < http://thes.bncf.firenze.sbn.it/termine.php?id=5401\&menuR=2\&menuS=2>

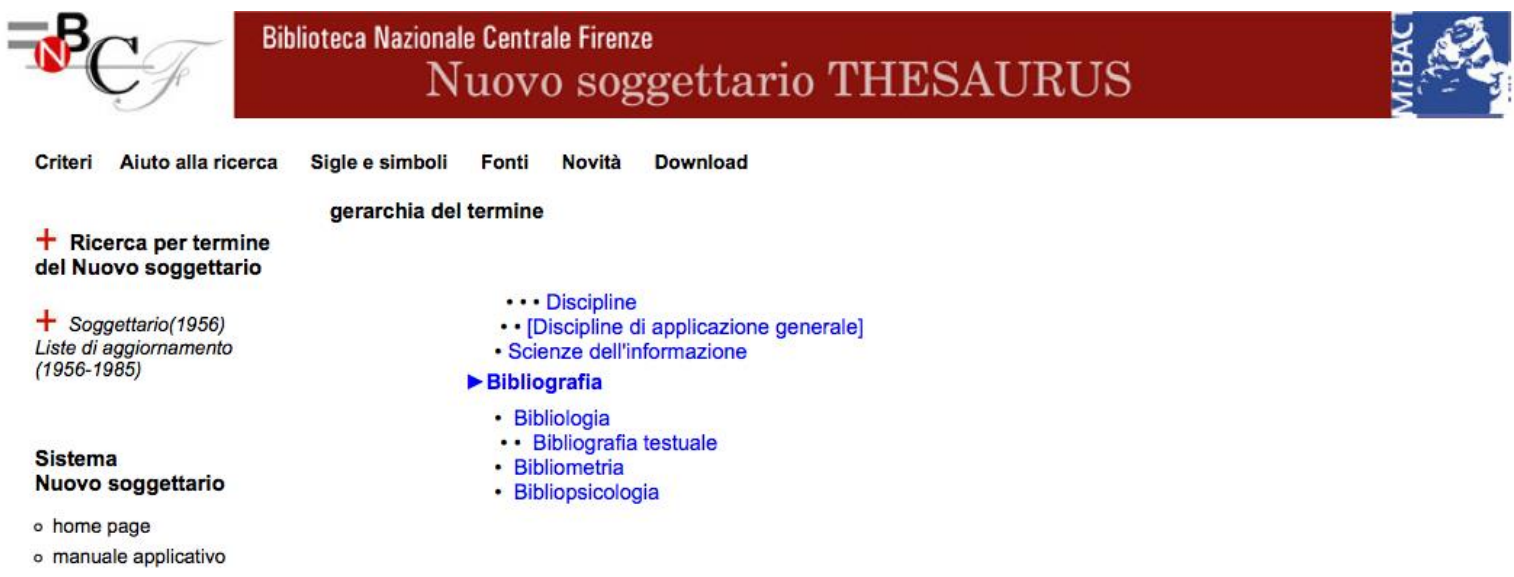

FIGURA 12 - Gerarchia del termine Bibliografia

Disponíbile: <http://thes.bncf.firenze.sbn.it/gerarchia.php?id=5401\&menuR=2\&menuS=2>

InCID: R. Ci. Inf. e Doc., Ribeirão Preto, v. 7, n. esp., p. 99-133, ago. 2016. 
Natura ed origini della Bibliografia: una prospettiva disciplinare per la contemporaneità

QUADRO 1 - Sigle delle relazioni semantiche utilizzate nel Thesaurus do Nuovo Soggettario

\begin{tabular}{|c|c|c|c|}
\hline Sigla & Sigla sciolta & Tipo di relazione & Significato \\
\hline BT & $\begin{array}{l}\text { Broader term } \\
\text { (Termine generico) }\end{array}$ & $\begin{array}{l}\text { Relazione } \\
\text { gerarchica }\end{array}$ & $\begin{array}{l}\text { relazione tra un termine } \\
\text { preferito e il termine } \\
\text { preferito immediatamente } \\
\text { sovraordinato (più } \\
\text { generale). La relazione } \\
\text { reciproca è NT }\end{array}$ \\
\hline HSEE & $\begin{array}{l}\text { Historical see } \\
\text { (Variante storica di) }\end{array}$ & $\begin{array}{l}\text { Legame di } \\
\text { variante storica } \\
\text { (legame non } \\
\text { standard) }\end{array}$ & $\begin{array}{l}\text { collegamento tra un } \\
\text { termine non più preferito } \\
\text { (variante storica) e il } \\
\text { nuovo termine preferito } \\
\text { che lo sostituisce. La } \\
\text { relazione reciproca è HSF }\end{array}$ \\
\hline HSF & $\begin{array}{l}\text { Historical seen for } \\
\text { (Ha come variante storica) }\end{array}$ & $\begin{array}{l}\text { Legame di } \\
\text { variante storica } \\
\text { (legame non } \\
\text { standard) }\end{array}$ & $\begin{array}{l}\text { collegamento tra un } \\
\text { termine preferito e una } \\
\text { sua variante storica. La } \\
\text { relazione reciproca è } \\
\text { HSEE }\end{array}$ \\
\hline NT & $\begin{array}{l}\text { Narrower term } \\
\text { (Termine specifico) }\end{array}$ & $\begin{array}{l}\text { Relazione } \\
\text { gerarchica }\end{array}$ & $\begin{array}{l}\text { relazione tra un termine } \\
\text { preferito e il termine } \\
\text { preferito immediatamente } \\
\text { subordinato (più } \\
\text { specifico). La relazione } \\
\text { reciproca è BT }\end{array}$ \\
\hline RT & $\begin{array}{l}\text { Related term } \\
\text { (Termine associato) }\end{array}$ & $\begin{array}{l}\text { Relazione } \\
\text { associativa }\end{array}$ & $\begin{array}{l}\text { relazione reciproca tra } \\
\text { due termini correlati }\end{array}$ \\
\hline SNR & Scope Note Reference & $\begin{array}{l}\text { Legame di } \\
\text { citazione nella } \\
\text { nota d'ambito }\end{array}$ & $\begin{array}{l}\text { collegamento tra un } \\
\text { termine non preferito } \\
\text { citato nella nota d'ambito } \\
\text { di un termine preferito e il }\end{array}$ \\
\hline
\end{tabular}


Fiammetta Sabba

\begin{tabular}{|c|c|c|c|}
\hline & & $\begin{array}{l}\text { (legame non } \\
\text { standard) }\end{array}$ & $\begin{array}{l}\text { termine preferito stesso. } \\
\text { La relazione reciproca è } \\
\text { SNX }\end{array}$ \\
\hline SNX & Is referenced in Scope Note & $\begin{array}{l}\text { Legame di } \\
\text { citazione nella } \\
\text { nota d'ambito } \\
\text { (legame non } \\
\text { standard) }\end{array}$ & $\begin{array}{l}\text { collegamento tra un } \\
\text { termine preferito e un } \\
\text { termine non preferito } \\
\text { citato nella sua nota } \\
\text { d'ambito. La relazione } \\
\text { reciproca è SNR }\end{array}$ \\
\hline TT & $\begin{array}{l}\text { Top Term } \\
\text { (Termine apicale) }\end{array}$ & $\begin{array}{l}\text { Relazione } \\
\text { gerarchica con il } \\
\text { termine apicale }\end{array}$ & $\begin{array}{l}\text { relazione tra un termine } \\
\text { preferito e il termine } \\
\text { apicale della sua gerarchia }\end{array}$ \\
\hline UF & $\begin{array}{l}\text { Used for } \\
\text { (Usato per) }\end{array}$ & $\begin{array}{l}\text { Relazione di } \\
\text { equivalenza }\end{array}$ & $\begin{array}{l}\text { relazione tra un termine } \\
\text { preferito e un suo } \\
\text { sinonimo o quasi- } \\
\text { sinonimo non preferito } \\
\text { (used for). La relazione } \\
\text { reciproca è USE }\end{array}$ \\
\hline $\mathrm{UF}+$ & $\begin{array}{l}\text { Used for }+ \\
\text { (È nel termine composto non preferito) }\end{array}$ & $\begin{array}{l}\text { Legame di } \\
\text { scomposizione }\end{array}$ & $\begin{array}{l}\text { collegamento tra i termini } \\
\text { preferiti derivanti dalla } \\
\text { scomposizione di un } \\
\text { termine composto non più } \\
\text { accettato e il composto } \\
\text { stesso. La relazione } \\
\text { reciproca è USE+ }\end{array}$ \\
\hline USE & Use (Usare) & $\begin{array}{l}\text { Relazione di } \\
\text { equivalenza }\end{array}$ & $\begin{array}{l}\text { relazione tra un termine } \\
\text { non preferito e il termine } \\
\text { preferito (use). La } \\
\text { relazione reciproca è UF }\end{array}$ \\
\hline USE+ & $\begin{array}{l}\text { Use }+ \\
\text { (Scomposto in) }\end{array}$ & $\begin{array}{l}\text { Legame di } \\
\text { scomposizione }\end{array}$ & $\begin{array}{l}\text { collegamento tra un } \\
\text { termine composto non più } \\
\text { accettato e i termini }\end{array}$ \\
\hline
\end{tabular}

InCID: R. Ci. Inf. e Doc., Ribeirão Preto, v. 7, n. esp., p. 99-133, ago. 2016. 


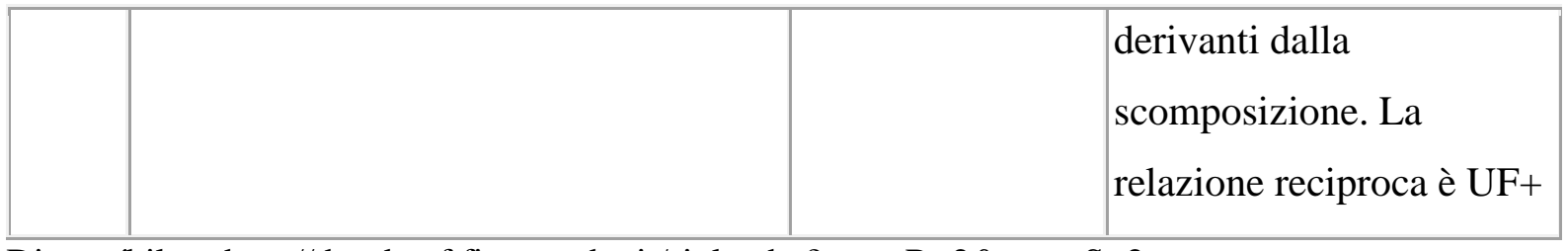

Disponíbile: <http://thes.bncf.firenze.sbn.it/sigle.php?menuR=2\&menuS=2>

\section{Conclusione: quanto Gesner è attuale? Quanto è utile conoscere la sua opera nel presente documentario del web?}

Se mi si chiede quale è la attualità del progetto bibliografico gesneriano, rispondo: il principio da cui si mosse, registrare il patrimonio scritto, classificarlo, e identificare ciò che era ancora inedito e quindi non reso pubblico.

Non è forse ciò che abbiamo continuato a fare nei cinque secoli successivi? E non è la stessa ansia che ci muove oggi ad interrogarci sui documenti, sulle biblioteche, e sugli archivi digitali?

Come possiamo reperire quanto è stato pubblicato in formato digitale o è disponibile digitalizzato nel web? Come possiamo organizzarlo in forma classificata? Quali sono le mappe scientifiche e documentarie che possono farci da bussola? Come possiamo operare sul web una ricerca semantica? Come possiamo capire cosa è presente e cosa ancora non lo è?

Immaginiamo il caos che la stampa aveva imposto agli eruditi del Cinquecento, e riflettiamo sul caos informativo di oggi: sulla ridondanza di dati, sulla mancanza di un 'luogo preciso' dove cercare, sulla necessità di poter interrogare un sistema ricevendo risposte pertinenti non dal punto di vista linguistico ma da quello semantico per non doverci fare strada nel web come nella foresta con un machete. La ricerca per parole nel web è ormai insufficiente perché a causa della mole delle informazioni che vi sono presenti, l'utente è costretto a filtrare e interpretare $\mathrm{i}$ risultati, a fare attenzione alla sinonimia, a cercare tra i risultati risposte pertinenti per contenuto. Ed ecco sono delle 'pandectae semantiche' che andiamo cercando!

Ad oggi sono possibili, anche se non tutti accessibili al volgo non specialistico, tre tipi di ricerca: la prima è di tipo terminologico e con il linguaggio term-based, tipici dell'Information retrieval (IR); la seconda è quella di tipo semantico, sviluppata soprattutto con il linguaggio dei linked data; ed infine quella di tipo semiotico con linguaggio content-based, sperimentati dal Multimedia information retrieval (MIR). 
Il tipo di ricerca che in questo contesto bibliografico e documentario più ci interessa è il secondo, ossia quello semantico, che opera tramite le corrette connessioni tra dati controllati alla base, quindi certificati e dedeferenziati tramite vocabolari, tassonomie, tesauri, ontologie gestiti da esperti. Le ontologie in particolare stanno diventando fondamentali per arginare l'overload informativo e per dare vita ad un substrato catalografico nuovo, che sia predisposto per fare parte del web.

Come sappiamo le biblioteche e il loro contenuto informativo non emergono che raramente nei risultati dei motori di ricerca, sia per la mancata applicazione della tecnologia informatica che lo rende possibile, sia perché fino a 5 o 6 anni fa si riteneva forse incosciamente necessario tenere separata la realtà bibliotecaria dal generalismo del web. Se questo aveva un fondamento, adesso non è più una considerazione valevole, almeno per il fatto che anche chi voglia raggiungere risposte di ambito bibliografico e quindi di pertinenza bibliotecaria, passa innanzitutto dal web, abituato ormai a un tipo di interrogazione semplificata, immediata e "nautica", e alle risposte dei siti commerciali di editori e librerie antiquarie.

Gli stessi OPAC sono costosi tenuto conto delle tecnologie disponibili e poco efficaci rispetto alle aspettative dei lettori abituati a percorsi via via scoperti grazie alle relazioni tra le entità. Perciò gli OPAC dovranno venire riadattati anche per quanto riguarda l'ordine degli elementi nell'interfaccia di ricerca, e dotati di raffinazione automatica dei risultati. Si tratta di possibilità già esistenti, intendiamoci, ma nel contesto bibliotecario hanno urgenza di essere applicate; e se le biblioteche vogliono infatti continuare a far parte della vita quotidiana della nostra società, devono poter 'penetrare' il web, invadere la curiosità dei cibernauti, ed imporsi alla loro quotidianità euristica.

Torniamo alla tecnologia dei linked data che sarà tra gli attori di questo cambiamento auspicato. Le biblioteche, come gli altri istituti culturali che sono gli archivi ed i musei, dovranno iniziare a lavorare in questa prospettiva servendosi delle numerosissime ontologie specialistiche: ad esempio quelle per la descrizione bibliografica e archivistica (ISBD, FRBR, RDA, OAD), per quella degli elementi di autorità (FRAD, FRSAD, LCSH), dei luoghi geografici (GeoNames e FAO Geopolitical Ontologies), dei vari diritti (Creative Commons Rights Expression Language), per le citazioni (BIBO, SPAR), per i metadati di conservazione, per i metadati di dataset ecc.

Le ricche possibilità metodologiche e repertoriali che la tecnologia ci offre, e delle quali abbiamo appena fornito degli esempi, ci fanno constatare come i momenti critici per la InCID: R. Ci. Inf. e Doc., Ribeirão Preto, v. 7, n. esp., p. 99-133, ago. 2016. 
documentazione siano quelli imposti dall'avvento appunto di nuove tecnologie, che nascono sempre in risposta alla necessità di aumentare il numero di persone raggiunte, cercando di annientare gli ostacoli del tempo e dello spazio. Ottenuto lo strumento, realizzata la possibilità, poi ci si trova a dover dominare gli eccessi ed il caos che le nuove situazioni generano. La ricerca di organizzazione ha le stesse premesse ogni volta, gli stessi fini, e le stesse logiche umane applicate.

Gesner è attuale dunque, anzi direi straordinariamente attuale. Con le dovute proporzioni di complessità gestionale, ciò che è riuscito a lui da solo, non sempre riesce altrettanto bene oggi ai nostri numerosi gruppi di lavoro e di ricerca. Dominare l'informazione, organizzare la conoscenza, arrivare ad una "ecologia informativa" è in questo momento la necessità più pressante e l'ambito più occupato dalle menti del nostro settore. Forse potrà appunto aiutarci la possibilità di riuso dei dati e la filosofia di responsabilità culturale proposta dallo standard di contenuto RDA (Resource Description and Access) con l'ausilio della tecnologia dei Linked data. Quelle linee guida e quella tecnologia potrebbero divenire la chiave per la costituzione di un web già comunemente chiamato, forse un po' troppo frettolosamente, 'semantico', nel senso che i legami creati tra gli elementi descrittivi dei documenti (ossia dei dati forniti in modo completo, esaustivo, e identificativo-dedeferenziante) saranno certificati, opportuni, congrui nel significato, e non casuali. Il web semantico corrisponderà così all'ambiente in cui le risorse pubblicate sono associate a informazioni e metadati che ne specificano il contesto semantico in un formato adatto all'elaborazione (ricerca) automatica. Le macchine dunque potranno accedere a un insieme strutturato di informazioni che consente loro di riutilizzare i dati in nuove forme, basate su regole di inferenza.

In questo contesto le biblioteche avranno ancora il loro gioco nel campo della scienza e dell'informazione documentaria come istituti culturali che, con gli archivi e i musei, dovranno occuparsi della conservazione e della sistematizzazione del patrimonio culturale, rendendone possibile ed agevole la visione e la fruizione, il pubblico godimento, sia estetico che scientifico. Questa partecipazione sarà fondamentale perché contrasterà la massa informativa del web incontrollata, superficiale e inattendibile, ed anche caotica e non organizzata, e che pertanto lascia sommersa e dispersa una buona parte delle informazioni e dei documenti rilevanti e qualificati.

La Bibliografia in conclusione non è solo una disciplina e una scienza, ma una filosofia di approccio responsabile, consapevole e strutturato all'universo dei documenti per garantire la 
permanenza della civiltà.

La Bibliografia attraverso le biblioteche affermerà ancora la sua missione in un futuro apparentemente lontano dal passato in cui collochiamo Gesner, ma forse, come lo stesso Gesner riterrebbe secondo la visione zwingliana che lo animava, in un futuro che col passato e il presente sono un unico momento dell'umanità.

\section{Referências}

\section{Per approfondire sulla epistemologia della Bibliografia}

BERNSTEIN, Lawrence F. The bibliography of music in Conrad Gesner's Pandectae (1548). Acta Musicologica, v. 45, fasc. 1, jan./jun.1973. pp. 119-163. Disponível em: <http://www.jstor.org/stable/932225>. Acesso em: 15 maio 2015.

BLAIR, Ann. Reading strategies for coping with information overload ca.1550-1700. Journal of the history of ideas, v. 64, n. 1, p.11-28, 2003.

EADEM. Un clamoroso plagio bibliografico alla metà del cinquecento. Bibliotheca, v. 4, n. 1, p. 279-285, 2005.

JASENAS, Michael. A history of the bibliography of philosophy. New York; Georg Olms verlag Hildesheim. 1973.

NELLES, Paul. Reading and memory in the universal library: Conrad Gesner and the renaissance book. In: BEECHER, Donald ; WILLIAMS, Grant (ed.). Ars reminiscendi: mind and memory in Renaissance culture. Toronto: Centre for Reformation and Renaissance Studies, 2009.

ROSENBERG, Daniel. Early Modern Information Overload. Journal of the History of Ideas, v. 64, n. 1, p. 1-9, January 2003.

SABBA, Fiammetta. "Documenti" e "Monumenti": un contributo alla epistemologia bibliografica. Accademie e Biblioteche d'Italia, v. 3/4, p. 11-19, 2004. Indici bibliografici. Bibliotheca, v. 5, n. 1, p.78-86, 2006.

SABBA, Fiammetta (Ed.). Le biblioteche private come paradigma bibliográfico: atti del Convegno Internazionale, Roma, Tempio di Ariano, 10-12 ottobre 2007. Roma: Bulzoni, 2008. 524 p. (Il Bibliotecario, 20).

. Introduzione. In: MINISTERO PER I BENI E LE ATTIVITÀ CULTURALI BIBLIOTECA ANGELICA. Indice dei nomi degli Autori dei manoscritti in scrittura latina della Biblioteca Angelica di Roma. Roma: Istituto Poligrafico e Zecca dello Stato, Libreria dello Stato, 2009. (Collezione "Indici e Cataloghi"). 
SERRAI, Alfredo. Analecta libraria: temi di critica bibliografica e di storia bibliotecaria. Roma: Bulzoni, 2000.

Biblioteconomia come scienza: introduzione ai problemi e alla metodologia. Firenze: Olschki, 1973.

Breve storia delle biblioteche in Italia. Milano: Sylvestre Bonnard, 2006.

Olschki, 1977.

Le classificazioni: idee e materiali per una teoria e per una storia. Firenze:

Il cimento della bibliografia. Milano: S. Bonnard, 2001.

Dai loci communes alla bibliometria. Roma: Bulzoni, 1984.

Flosculi bibliographici. Roma: Bulzoni, 2001.

Guida alla biblioteconomia. Firenze: Sansoni, 1983.

Dalla informazione alla bibliografia: la professione bibliotecaria. Milano: Bibliografica. 1984.

. Natura elementi e origine della bibliografia in quanto mappa del sapere e delle lettere. Roma: Bulzoni, 2010.

Ricerche di biblioteconomia e di bibliografia. Firenze: La nuova Italia, 1983.

Storia della bibliografia: Roma: Bulzoni, 1988-2011. (11 volumes em 13 tomos: I-IX; X/1-2; XI/1-2): 1. Bibliografia e Cabala; Le enciclopedie rinascimentali (1). - 2. Le enciclopedie rinascimentali (2); Bibliografi universali. - 3. Vicende e ammaestramenti della 'Historia literaria'. - 4. Cataloghi a stampa; Bibliografie teologiche; Bibliografie filosofiche; A. Possevino. - 5. Trattatistica biblioteconomica. - 6. La maturità disciplinare. - 7. Storia e critica della catalogazione bibliografica / a cura di Gabriella Miggiano. - 8. Sistemi e tassonomie / a cura di Marco Menato. - 9. Manualistica, didattica e riforme nel sec. XVIII. 10/I-II. Specializzazione e pragmatismo: i nuovi cardini della attività bibliografica. - 11/I-II. Indici volumi I-X.

SERRAI, Alfredo; SABBA, Fiammetta. Profilo di Storia della bibliografia. Milano: Edizioni Sylvestre Bonnard, 2005.

* Numerosi articoli ed interventi nei periodici bibliografici: «ll Bibliotecario» Roma, Bulzoni Editore (1984-1999, 2008-2011); «Bibliotheca, Rivista di studi bibliografici» Milano, Sylvestre Bonnard (2002-2007); «Bibliothecae.it» Perugia, Morlacchi Editore (2012-2015, e dal 2016 pubblicata in open access nella piattaforma ALMA DL dell'Alma Mater Studiorum di Bologna). 
CANFORA, Luciano. Il Fozio ritrovato: Juan de Mariana e André Schott. Con l'inedita Epitome della Biblioteca di Fozio ed una raccolta di documenti a cura di Giuseppe Solaro. Appendici di Renata Roncali, Niccolò Zorzi, Margherita Losacco, Luciano Canfora. Bari: Edizioni Dedalo, 2001.

CECCARELLI, Maria Grazia. il fondo "Tigurino" della Biblioteca Passionei all'Angelica. Il Bibliotecario, v. 9, p. 93-132, 1986.

COCHETTI, Maria. Repertori bibliografici del cinquecento. Roma: Bulzoni, 1987.

DAVIS, Margaret Daly. Conrad Gesner: Pandectarum, sive Partitionum universalium ... libri XXI (Zürich 1548). Auszüge zur Kunstgeschichte und Altertumswissenschaft / Excerpted bibliographies for the history of art and the study of antiquity. Fontes. Quellen und Dockumente zur Kunst 1350-1750. (1 nov. 2007). Disponível em: <http://archiv.ub.uniheidelberg.de/artdok/378/1/Daly_Davis_Fontes1.pdf>

FISCHER, Hans; PETIT, Georges, STAEDTKE, Joachim, STEIGER, Rudolf, ZOLLER, Heinrich. Conrad Gessner, 1516-1565: Universalgelehrter, Naturforscher, Arzt. Zürich, 1967.

FRYDE, Edmund B. Humanism and Renaissance Historiography. London: Hambledon Press, 1983.

GERMANN, Martin. Die reformierte Stiftsbibliothek am Grossmünster Zürich im 1. Jahrhundert und die Anfänge der neuzeitlichen Bibliographie. Rekonstruktion des Buchbestandes und seiner Herkunft, der Bücheraufstellung un des Bibliotheksraumes. Mit Edition des Inventars 1532/1551 von Conrad Pellikan. Wiesbaden: Harrassowitz, 1994.

HANHART, Johannes. Conrad Gessner. Ein Beytrag zur Geschichte des wissenschaftlichen Strebens und der Glaubensverbesserung im 16ten Jahrhundert. Winterthur: in der Steinerischen Buchhandlung, 1824.

HARTMANN, Alfred; JENNY, Beat Rudolf. Die Amerbachkorrespondenz. VI. Band. Die Briefe aus den Jahren 1544-1547. Basel: Verlag der Universitätbibliothek, 1967.

HOBSON, Anthony. The "Iter Italicum" of Jean Matal. In: STUDIES in the book trade in honour of Graham Pollard. Oxford: Oxford Bibliographical Society, 1975. p. 33-61. (Oxford Bibliographical Society Publications, 18.).

HOBSON, Anthony. Renaissance book collecting: Jean Grolier and Diego Hurtado de Mendoza, their books and bindings. Cambridge, University Press: 1999.

IRIGOIN, Jean. Les ambassadeurs a Venise et le commerce des manuscrits grecs dans le années 1540-1550. In: BECK, Hans-Georg; MANOUSSACAS Manoussos, PERTUSI, Agostino. Venezia centro di mediazione tra Oriente e Occidente (secoli XV-XVI): aspetti e problemi. Firenze: Leo S. Olschki, 1977. V. 2. p.399-415. 
JENNY, Beat Rudolf. Arlenius in Basel. Basler Zeitschrift für Geschichte und Altertumskunde, 64 (1964), p. 5-45.

LEU, Urs B. Conrad Gesner als theologe: ein Beitrag zur Zürcher Geistesgeschiochte des 16. Jahrhunderts. Bern: P. Lang, 1990.

LEU, Urs B.; KELLER, Raffael; WEIDWANN, Sandra. Conrad Gessner's private library. Leiden-Boston: Brill, 2008.

SABBA, Fiammmeta. La Biblioteca di Diego Hurtado de Mendoza nella "Bibliotheca Universalis" di Conrad Gesner. Bibliotheca, v. 6, n. 2, p. 93-112, 2007.

La 'Bibliotheca Universalis' di Conrad Gesner: monumento della cultura europea. Roma: Bulzoni, 2012.

Gli scrittori italiani di Medicina nella 'Bibliotheca Universalis' di Conrad Gesner", Bibliologia, v. 8, p. 39-62, 2013.

SERRAI, Alfredo. Conrad Gesner. Roma: Bulzoni, 1990.

. Domenico Passionei e la sua Biblioteca. Milano: Sylvestre Bonnard, 2004.

. I 'Pandectae’ di Conrad Gesner. Bibliotheca, v. 1, p. 11-37, 2007.

. Una scoperta sensazionale all'Angelica: da Gesner a Passionei e

Scheuchzer. Il Bibliotecario, v. 7/8, p. 81-103, 1986.

STEIGER, Rudolf. Erschließung des Conrad-Geßner-Materials der Zentralbibliothek Zürich, Gessnerus, v. 25, p. 29-64, 1968.

WELLISH, Hans. Conrad Gessner: a bio-bibliography. Zürich, 1984.

Alcuni titoli di autori italiani per approfondire i temi trattati legati alla contemporaneità catalografica e dell'information retrieval

BIAGETTI, Maria Teresa. Nuove funzionalità degli OPAC e relevance ranking. Bollettino AIB, v. 50, n. 4, p. 339-356, 2010.

. Sviluppi e trasformazioni delle biblioteche digitali: dai repositories di testi alle semantic digital libraries. AIB studi, v. 54, n. 1, p. 11-34, 2014. DOI: 10.2426/aibstudi-9955. gennaio/aprile

BIANCHINI, Carlo; GUERRINI, Mauro. Introduzione a RDA. Milano: Bibliografica, 2014. [Recensione di Fiammetta Sabba: Bibliothecae.it. Rivista semestrale di studi, v. 3, n. 2, p. 239-240, 2014] 
GUERRINI, Mauro; POSSEMATO, Tiziana. Linked data per biblioteche, archivi e musei. Milano: Editrice Bibliografica, 2015 [Recensione di Fiammetta Sabba: Bibliothecae.it. Rivista semestrale di studi, v. 5, n. 1, 2016. No prelo].

RAIELI, Roberto; INNOCENTI, PERLA (Ed.). Multimedia information retrieval: metodologie ed esperienze internazionali di content-based retrieval per l'informazione e la documentazione. Premissa di Lucia Mafferi. Roma: AIDA, 2004.

RAIELI, Roberto. Così lontani, così vicini: i possibili alleati delle biblioteche nelle sfide per la trasmissione delle conoscenze. AIB studi, v. 53, n. 1, p.5-8, 2013.

. Multimedia Digital Libraries Handling: the Organic MMIR Perspective, in Bridging between Cultural Heritage Institutions. In: ITALIAN RESEARCH CONFERENCE ON DIGITAL LIBRARIES, 9., 2013. Proceedings... BerlinHeidelberg: Springer, 2014. p. 171-186. v. 385.

2013.

. Multimedia Information Retrieval: theory and techniques. Oxford: Chandos,

Nuovi metodi di gestione dei documenti multimediali: principi e pratica del MultiMedia Information Retrieval. Milano: Bibliografica, 2010.

. The semantic hole: enthusiasm and caution around the MultiMedia Information Retrieval, Knowledge Organization, v. 39, n. 1, p.13-22, 2012.

SABBA, Fiammetta. Il digitale deve confrontarsi con la Bibliografia. Bibliothecae.it., v. 2, n. 1, p. 281-289, 2013.

La Biblioteca digitale tra risorsa e aspirazione del bibliografo. Atti del Convegno

'Noetica versus informatica': le nuove strutture della conoscenza scientifica' (19-20 novembre 2013). Roma-Firenze, CCIAA-Olschki, 2015. p. 217-229 (più 'Nota del curatore' a p. XI).

Cantiere documentazione. Bibliothecae.it, v. 4, n. 1, p.147-161, 2015.

SABBA, Fiammetta (Ed.). Noetica versus informatica: le nuove strutture della comunicazione scientifica. Atti del convegno internazionale, 19-20 novembre 2013. Firenze: Leo S. Olschki, 2015. 